\title{
ON WEIGHT MODULES OF ALGEBRAS OF TWISTED DIFFERENTIAL OPERATORS ON THE PROJECTIVE SPACE
}

\author{
DIMITAR GRANTCHAROV AND VERA SERGANOVA
}

\begin{abstract}
We classify blocks of categories of weight and generalized weight modules of algebras of twisted differential operators on $\mathbb{P}^{n}$. Necessary and sufficient conditions for these blocks to be tame and proofs that some of the blocks are Koszul are provided. We also establish equivalences of categories between these blocks and categories of bounded and generalized bounded weight $\mathfrak{s l}(n+1)$-modules in the cases of nonintegral and singular central character.
\end{abstract}

2000 MSC: 17B10

Keywords and phrases: Lie algebra, indecomposable representations, quiver, weight modules, twisted differential operators

\section{INTRODUCTION}

Algebras of twisted differential operators play important role in modern representation theory. One fundamental application of these algebras is the equivalence of categories of modules over a complex semsimple Lie algebra and modules of global sections of algebras of twisted differential operators established by Beilinson and Bernstein, [3]. In this paper we initiate the study of generalized weight modules of such algebras, i.e. modules that are locally finite with respect to a maximal commutative subalgebra.

The first step in our study is to look at the category of generalized weight modules over the Weyl algebra $\mathcal{D}(n+1)$. We classify the blocks of this category and prove that these blocks are equivalent to the category of locally nilpotent $G$-graded representations of the polynomial algebra $\mathbb{C}\left[z_{0}, \ldots, z_{n}\right]$, where $G$ is the direct product of several copies of $\mathbb{Z}_{2}$. Weight modules of the Weyl algebra were studied by Bavula, Bekkert, Benkart, Futorny, among others, [1], 3], [5], [17].

The original motivation of this study comes from the problem of classifying blocks of generalized weight modules with bounded set of weight multiplicities over the Lie algebra $\mathfrak{s l}(n+1)$. Such modules are called generalized bounded modules. The natural action of $S L(n+1)$ on the affine space $\mathbb{A}^{n+1}$ induces a homomorphism $\psi$ : $U(\mathfrak{s l}(n+1)) \rightarrow \mathcal{D}(n+1)$. The image of this homomorphism lies in the subalgebra $\mathcal{D}^{E} \subset \mathcal{D}(n+1)$ of operators commuting with the Euler vector field $E$. In many cases

D.G is supported by NSA grant H98230-13-1-0245; V.S. is supported by NSF grant 0901554. 
generalized bounded weight $\mathfrak{s l}(n+1)$-modules can be obtained from $\mathcal{D}^{E}$-modules via $\psi$. If $Z$ is the center of $U(\mathfrak{s l}(n+1))$, then $\psi(Z)$ lies in the subalgebra of $\mathcal{D}(n+1)$ generated by $E$. Hence, all $\mathfrak{s l}(n+1)$-modules with a fixed central character come from $\mathcal{D}^{E} /(E-a)$-modules for a fixed $a \in \mathbb{C}$. Note that $\mathcal{D}^{a}=\mathcal{D}^{E} /(E-a)$ is nothing else but the algebra of global section of twisted differential operators on the projective space $\mathbb{P}^{n}$.

It is not hard to see that any indecomposable generalized weight module of finite length admits a generalized central character. Therefore, it is important to study generalized weight modules over the ring $\widetilde{\mathcal{D}}^{a}=\lim \mathcal{D}^{E} /(E-a)^{n}$, which itself can be described as the ring of formal deformations of $\overleftarrow{\mathcal{D}^{a}}$. We classify the blocks of generalized weight $\widetilde{\mathcal{D}}^{a}$-modules in terms of quivers with relations. Then we prove that the category of generalized weight $\mathfrak{s l}(n+1)$-modules with bounded weight multiplicities that admit non-integral or singular integral generalized central character is equivalent to the category of generalized weight $\widetilde{\mathcal{D}}^{a}$-modules for a suitable choice of $a$. The proof uses variations of the twisted localization construction originally introduced in [19] and the classification of generalized weight cuspidal blocks, see [20]. We also obtain all classification results and equivalences of categories mentioned above in the case of weight modules - those for which the action of the corresponding commutative subalgebra is semisimple.

Let us mention that the above result for a non-integral or singular integral central character is essentially a consequence of the fact that in this case all simple bounded weight modules with the same central character are annihilated by the same primitive ideal. If the central character is regular integral, then we have $n+1$ primitive ideals representing annihilators of the modules in the corresponding block. This case was solved in [18] and in [20] for cuspidal weight modules and for generalized cuspidal weight modules, respectively. The case of general bounded modules with regular integral central character remains open in general.

The paper is organized as follows. In Section 3 we collect some important definitions and facts for generalized weight modules of $\mathfrak{s l}(n+1)$ and $\widetilde{\mathcal{D}}^{a}$. The quivers and the corresponding algebras that appear in the classification results are introduced in Section 4. The study of generalized weight modules of $\mathcal{D}(n+1)$ is presented in Section 5 , while the next section is devoted to the study of these modules over $\widetilde{\mathcal{D}}^{a}$. The twisted localization construction is presented in Section 7 and the main results on the equivalence of categories are included in Section 8. Some useful commutative diagrams of functors are collected in the Appendix.

Acknowledgements. The authors would like to thank to V. Bekkert for the useful information provided for certain quivers. We also thank L. Hille and A. Polischuk for the fruitful discussions. 


\section{INDEX OF NOTATIONS}

Below we list some notations that are frequently used in the paper under the section number they are introduced.

3.1 supp, $M^{\lambda}, M^{(\lambda)}$.

$3.2 \mathcal{D}(n+1), \mathcal{D},(\mathcal{D}, \mathcal{H})-\bmod ,{ }^{\mathrm{b}}(\mathcal{D}, \mathcal{H})-\bmod ,(\mathcal{D}, \mathcal{H})_{\nu}-\bmod ,{ }^{\mathrm{b}}(\mathcal{D}, \mathcal{H})_{\nu}-\bmod$

$3.3|\nu|, E,\left(\mathcal{D}^{E}, \mathcal{H}\right)^{a}-\bmod ,\left(\mathcal{D}^{E}, \mathcal{H}\right)_{\nu}^{a}-\bmod ,{ }_{\mathrm{s}}\left(\mathcal{D}^{E}, \mathcal{H}\right)^{a}-\bmod ,{ }_{\mathrm{s}}^{\mathrm{b}}\left(\mathcal{D}^{E}, \mathcal{H}\right)^{a}-\bmod ,{ }_{\mathrm{s}}\left(\mathcal{D}^{E}, \mathcal{H}\right)_{\nu^{-}}$ $\bmod ,{ }_{\mathrm{s}}^{\mathrm{b}}\left(\mathcal{D}^{E}, \mathcal{H}\right)_{\nu}^{a}$-mod.

$3.4 \gamma, \mathcal{B}, \mathcal{G B}, \overline{\mathcal{B}}, \overline{\mathcal{G B}}, \mathcal{B}_{\nu}, \mathcal{G B}{ }_{\nu}, \mathcal{B}^{\lambda}, \mathcal{G} \mathcal{B}^{\lambda}, \mathcal{B}_{\nu}^{\lambda}, \mathcal{G} \mathcal{B}_{\nu}^{\lambda}, \overline{\mathcal{B}}_{\nu}^{\lambda}, \overline{\mathcal{G B}}_{\nu}^{\lambda}$

$5.3 S_{\mathcal{H}^{\prime}}, \mathcal{B}_{\nu}, \mathcal{B}(k)$.

$4.1 C(k), A(k), A^{\prime}(k), A^{\prime \prime}(k), B(k), B^{\prime}(k), B^{\prime \prime}(k)$.

$5.2 \mathcal{F}_{\nu}, \mathcal{F}_{\nu}^{\log }, \sigma_{J}, \mathcal{I}_{\nu}(J), \mathcal{F}_{\nu}^{\log }(J), \operatorname{Int}(\nu), \mathcal{P}(\nu), \mathcal{R}_{\nu}, \mathcal{A}_{\nu}, G_{k}$.

$6.1 \Gamma_{a}, \Phi, \pi^{a}$.

$6.2 \mathcal{P}_{a}, \Phi^{\prime}, \mathcal{R}_{0}^{a}$

$7.1 D_{F}, \Theta_{F}^{\mathrm{x}}, \Theta_{F}^{\mathrm{x}}, D_{F}^{\mathrm{x}}$.

$7.3 D_{i}^{+}, D_{i}^{-}, D_{i}^{x,+}, D_{i}^{x,-}, D_{i, j}, D_{i, j}^{x}$.

$8.1 \psi, \Psi$.

$8.2 S_{\mathfrak{h}}$.

\section{BACKGROUND}

In this paper the ground field is $\mathbb{C}$. All vector spaces, algebras, and tensor products are assumed to be over $\mathbb{C}$ unless otherwise stated.

3.1. Categories of weight modules of associative algebras. Let $\mathcal{U}$ be a finitely generated associative unital algebra and $\mathcal{H} \subset \mathcal{U}$ be a commutative subalgebra. We assume in addition that $\mathcal{H}=\mathbb{C}\left[h_{0}, \ldots, h_{n}\right]=S(\mathfrak{h})$ is a polynomial algebra and ad $(h): \mathcal{U} \rightarrow \mathcal{U}$ is semisimple for all $h \in \mathfrak{h}$. In other words, we have a decomposition

$$
\mathcal{U}=\bigoplus_{\mu \in \mathfrak{h}^{*}} \mathcal{U}^{\mu}
$$

where

$$
\mathcal{U}^{\mu}=\{x \in \mathcal{U} \mid[h, x]=\mu(h) x, \forall h \in \mathfrak{h}\} .
$$

Let $Q=\mathbb{Z} \Delta_{\mathcal{U}}$ be the $\mathbb{Z}$-lattice in $\mathfrak{h}^{*}$ generated by $\Delta_{\mathcal{U}}=\left\{\mu \in \mathfrak{h}^{*} \mid U^{\mu} \neq 0\right\}$. We note that $Q$ is of finite rank since $\mathcal{U}$ is finitely generated. We also obviously have $U^{\mu} U^{\nu} \subset U^{\mu+\nu}$.

We call a $\mathcal{U}$-module $M$ a generalized weight $(\mathcal{U}, \mathcal{H})$-module if $M=\bigoplus_{\lambda \in \mathfrak{h}^{*}} M^{(\lambda)}$, where

$$
M^{(\lambda)}=\left\{m \in M \mid\left(h_{i}-\lambda\left(h_{i}\right) \mathrm{Id}\right)^{N} m=0 \text { for some } N>0 \text { and all } i=0, \ldots, n\right\} .
$$

We call $M^{(\lambda)}$ the generalized weight space of $M$ and $\operatorname{dim} M^{(\lambda)}$ the weight multiplicity of the weight $\lambda$. Note that

$$
\mathcal{U}^{\mu} M^{(\lambda)} \subset M^{(\mu+\lambda)}
$$


A generalized weight module $M$ is called a weight module if $M^{(\lambda)}=M^{\lambda}$, where

$$
M^{\lambda}=\left\{m \in M \mid\left(h_{i}-\lambda\left(h_{i}\right) \mathrm{Id}\right) m=0 \text { for all } i=0, \ldots, n\right\} .
$$

By $(\mathcal{U}, \mathcal{H})-\bmod$ and ${ }^{\mathrm{w}}(\mathcal{U}, \mathcal{H})-\bmod$ we denote the category of generalized weight modules and weight modules, respectively. Furthermore, by ${ }^{\mathrm{f}}(\mathcal{U}, \mathcal{H})$-mod $\operatorname{and}^{\mathrm{b}}(\mathcal{U}, \mathcal{H})$ mod we denote the subcategories of $(\mathcal{U}, \mathcal{H})$-mod consisting of modules with finite weight multiplicities and bounded set of weight multiplicities, respectively. $\operatorname{By}^{\text {wf }}(\mathcal{U}, \mathcal{H})$ $\bmod$ and ${ }^{\mathrm{wb}}(\mathcal{U}, \mathcal{H})$-mod we denote the subcategories of ${ }^{\mathrm{w}}(\mathcal{U}, \mathcal{H})-\bmod$ that are in ${ }^{\mathrm{f}}(\mathcal{U}, \mathcal{H})-\bmod$ and ${ }^{\mathrm{b}}(\mathcal{U}, \mathcal{H})-\bmod$, respectively.

For any module $M$ in $(\mathcal{U}, \mathcal{H})$-mod we set

$$
\operatorname{supp} M:=\left\{\lambda \in \mathfrak{h}^{*} \mid M^{(\lambda)} \neq 0\right\}
$$

to be the support of $M$. It is clear from (3.1) that $\operatorname{Ext}_{\mathcal{A}}^{1}(M, N)=0$ if $(\operatorname{supp} M-$ $\operatorname{supp} N) \cap Q=\emptyset$, where $\mathcal{A}$ is any of the categories of generalized weight modules or weight modules defined above. Then we have

$$
(\mathcal{U}, \mathcal{H})-\bmod =\bigoplus_{\bar{\mu} \in \mathfrak{h}^{*} / Q}(\mathcal{U}, \mathcal{H})_{\bar{\mu}}-\bmod
$$

where $(\mathcal{U}, \mathcal{H})_{\bar{\mu}}-\bmod$ denotes the subcategory of $(\mathcal{D}, \mathcal{H})$-mod consisting of modules $M$ with $\operatorname{supp} M \subset \bar{\mu}=\mu+Q$. We similarly define ${ }^{\mathrm{w}}(\mathcal{U}, \mathcal{H})_{\bar{\mu}}-\bmod ,{ }^{\mathrm{f}}(\mathcal{U}, \mathcal{H})_{\bar{\mu}}-\bmod$, ${ }^{\mathrm{b}}(\mathcal{U}, \mathcal{H})_{\bar{\mu}}-\bmod ,{ }^{\text {wf }}(\mathcal{U}, \mathcal{H})_{\bar{\mu}}-\bmod$, and ${ }^{\mathrm{wb}}(\mathcal{U}, \mathcal{H})_{\bar{\mu}}-\bmod$, and obtain the corresponding support composition where the direct summands are parametrized by elements of $\mathfrak{h}^{*} / Q$. With a slight abuse of notation, for $\mu \in \mathfrak{h}^{*}$ we set $(\mathcal{U}, \mathcal{H})_{\mu}-\bmod =$ $(\mathcal{U}, \mathcal{H})_{\bar{\mu}}-\bmod$, etc.

3.2. Weight $\mathcal{D}$-modules. Let $\mathcal{D}(n+1)$ be the Weyl algebra, i.e. the algebra of differential operators of the ring $\mathbb{C}\left[t_{0}, \ldots, t_{n}\right]$ and consider $\mathcal{U}=\mathcal{D}(n+1)$. When $n \geq 0$ is fixed, we use the notation $\mathcal{D}$ for $\mathcal{D}(n+1)$. Let $\mathcal{H}:=\mathbb{C}\left[t_{0} \partial_{0}, \ldots, t_{n} \partial_{n}\right]$. Then $\mathcal{H}$ is a maximal commutative subalgebra in $\mathcal{D}$. Note that the adjoint action of the abelian Lie subalgebra $\operatorname{Span}\left(t_{0} \partial_{0}, \ldots, t_{n} \partial_{n}\right)$ on $\mathcal{D}$ is semisimple. We identify $\mathbb{C}^{n+1}$ with the dual space of $\operatorname{Span}\left\{t_{0} \partial_{0}, \ldots, t_{n} \partial_{n}\right\}$, and fix $\left\{\varepsilon_{0}, \ldots, \varepsilon_{n}\right\}$ to be the standard basis of this space, i.e. $\varepsilon_{i}\left(t_{j} \partial_{j}\right)=\delta_{i j}$. Then $Q=\bigoplus_{i=0}^{n} \mathbb{Z} \varepsilon_{i}$ is identified with $\mathbb{Z}^{n+1}$, and

$$
\mathcal{D}=\bigoplus_{\mu \in \mathbb{Z}^{n+1}} \mathcal{D}^{\mu}
$$

Here $\mathcal{D}^{0}=\mathcal{H}$ and each $\mathcal{D}^{\mu}$ is a free left $\mathcal{H}$-module of rank 1 with generator $\prod_{\mu_{i} \geq 0} t_{i}^{\mu_{i}} \prod_{\mu_{j}<0} \partial_{j}^{-\mu_{j}}$.

Therefore ${ }^{\mathrm{b}}(\mathcal{D}, \mathcal{H})-\bmod ={ }^{\mathrm{f}}(\mathcal{D}, \mathcal{H})-\bmod$ and ${ }^{\mathrm{wb}}(\mathcal{D}, \mathcal{H})-\bmod ={ }^{\mathrm{wf}}(\mathcal{D}, \mathcal{H})-\bmod$. The latter category was studied in [5] and [17] and the former in [2]. 
The support of every $(\mathcal{D}, \mathcal{H})$-module will be considered as a subset of $\mathbb{C}^{n+1}$ and we have a natural decomposition

$$
(\mathcal{D}, \mathcal{H})-\bmod =\bigoplus_{\bar{\nu} \in \mathbb{C}^{n+1} / \mathbb{Z}^{n+1}}(\mathcal{D}, \mathcal{H})_{\bar{\nu}}-\bmod
$$

As before for $\nu \in \mathbb{C}^{n+1}$ we write $(\mathcal{D}, \mathcal{H})_{\nu}-\bmod =(\mathcal{D}, \mathcal{H})_{\bar{\nu}}-\bmod$. The same applies for the subcategories ${ }^{\mathrm{w}}(\mathcal{D}, \mathcal{H})_{\bar{\nu}}-\bmod ,{ }^{\mathrm{b}}(\mathcal{D}, \mathcal{H})_{\bar{\nu}}-\bmod ={ }^{\mathrm{f}}(\mathcal{D}, \mathcal{H})_{\bar{\nu}}-\bmod$, and ${ }^{\mathrm{wb}}(\mathcal{D}, \mathcal{H})_{\bar{\nu}}-\bmod ={ }^{\mathrm{wf}}(\mathcal{D}, \mathcal{H})_{\bar{\nu}}-\bmod$.

3.3. Weight $\mathcal{D}^{E}$-modules. In this subsection we assume $n \geq 1$. Let $E=\sum_{i=0}^{n} t_{i} \partial_{i}$ be the Euler vector field. Denote by $\mathcal{D}^{E}$ the centralizer of $E$ in $\mathcal{D}$. Note that $\mathcal{D}$ has a $\mathbb{Z}$-grading $\mathcal{D}=\bigoplus_{m \in \mathbb{Z}} \mathcal{D}^{m}$, where $\mathcal{D}^{m}=\{d \in \mathcal{D} \mid[E, d]=m d\}$. It is not hard to see that the center of $\mathcal{D}^{E}$ is generated by $E$. The quotient algebra $\mathcal{D}^{E} /(E-a)$ is the algebra of global sections of twisted differential operators on $\mathbb{P}^{n}$.

Let $a \in \mathbb{C}$, let $\left(\mathcal{D}^{E}, \mathcal{H}\right)^{a}$-mod be the category of generalized weight $\mathcal{D}^{E}$-modules with locally nilpotent action of $E-a$ and ${ }^{\mathrm{b}}\left(\mathcal{D}^{E}, \mathcal{H}\right)^{a}$-mod be the subcategory of ${ }^{\mathrm{b}}\left(\mathcal{D}^{E}, \mathcal{H}\right)^{a}$-mod consisting of modules with finite weight multiplicities. We have again a decomposition

$$
\left(\mathcal{D}^{E}, \mathcal{H}\right)^{a}-\bmod =\bigoplus_{|\nu|=a}\left(\mathcal{D}^{E}, \mathcal{H}\right)_{\nu}^{a}-\bmod
$$

where $\left(\mathcal{D}^{E}, \mathcal{H}\right)_{\nu}^{a}-\bmod$ is the subcategory of modules with support in $\nu+\sum_{i=0}^{n-1} \mathbb{Z}\left(\varepsilon_{i}-\right.$ $\left.\varepsilon_{i+1}\right)$ and $|\nu|:=\sum_{i=0}^{n} \nu_{i}$.

Let $\mathcal{H}^{\prime}$ be the subalgebra of $\mathcal{D}$ generated by $t_{i} \partial_{i}-t_{j} \partial_{j}$. We denote by ${ }_{\mathrm{s}}\left(\mathcal{D}^{E}, \mathcal{H}\right)$ $\bmod \left(\right.$ respectively, ${ }_{\mathrm{s}}^{\mathrm{b}}\left(\mathcal{D}^{E}, \mathcal{H}\right)$-mod) the the subcategory of $\left(\mathcal{D}^{E}, \mathcal{H}\right)$-mod (resp., the subcategory of ${ }^{\mathrm{b}}\left(\mathcal{D}^{E}, \mathcal{H}\right)$-mod) consisting of all modules semisimple over $\mathcal{H}^{\prime}$. Similarly we define the categories ${ }_{\mathrm{s}}\left(\mathcal{D}^{E}, \mathcal{H}\right)^{a}$-mod, ${ }_{\mathrm{s}}^{\mathrm{b}}\left(\mathcal{D}^{E}, \mathcal{H}\right)^{a}$-mod, ${ }_{\mathrm{s}}\left(\mathcal{D}^{E}, \mathcal{H}\right)_{\nu}^{a}$-mod, and ${ }_{\mathrm{s}}^{\mathrm{b}}\left(\mathcal{D}^{E}, \mathcal{H}\right)_{\nu}^{a}$-mod.

3.4. Weight $\mathfrak{s l}(n+1)$-modules. Let $\mathfrak{g}=\mathfrak{s l}(n+1)$ and $U=U(\mathfrak{g})$ be its universal enveloping algebra. We fix a Cartan subalgebra $\mathfrak{h}$ of $\mathfrak{g}$ and denote by $($,$) the Killing$ form on $\mathfrak{g}$. We apply the setting of $\$ 3.1$ with $\mathcal{U}=U$ and $\mathcal{H}=S(\mathfrak{h})$. We will use the following notation: $\mathcal{G B}={ }^{\mathrm{b}}(\mathcal{U}, \mathcal{H})-\bmod , \mathcal{B}={ }^{\mathrm{wb}}(\mathcal{U}, \mathcal{H})-\bmod , \mathcal{G B}_{\mu}=\mathcal{G B}_{\bar{\mu}}=$ $\mathrm{b}(\mathcal{U}, \mathcal{H})_{\bar{\mu}}-\bmod$, and $\mathcal{B}_{\mu}=\mathcal{B}_{\bar{\mu}}={ }^{\mathrm{wb}}(\mathcal{U}, \mathcal{H})_{\bar{\mu}}-\bmod$

A generalized weight module $M$ with finite weight multiplicities will be called a generalized cuspidal module if the elements of the root space $\mathfrak{g}^{\alpha}$ act injectively (and hence bijectively) on $M$ for all roots $\alpha$ of $\mathfrak{g}$. If $M$ is a weight cuspidal module we will call it simply cuspidal module. By $\mathcal{G C}$ and $\mathcal{C}$ we will denote the categories of generalized cuspidal and cuspidal modules, respectively, and the corresponding subcategories defined by the supports will be denoted by $\mathcal{G C}_{\bar{\mu}}$ and $\mathcal{C}_{\bar{\mu}}$. One should note that the simple objects of $\mathcal{B}$ and $\mathcal{G B}$ (as well as those of $\mathcal{C}$ and $\mathcal{G C}$ ) coincide.

The induced form on $\mathfrak{h}^{*}$ will be denoted by $\left(\right.$, , ) as well. We have that $\mathfrak{h}^{*}$ is identified with the subspace of $\mathbb{C}^{n+1}$ spanned by the simple roots $\varepsilon_{0}-\varepsilon_{1}, \ldots, \varepsilon_{n-1}-\varepsilon_{n}$. By $\gamma$ 
we denote the projection $\mathbb{C}^{n+1} \rightarrow \mathfrak{h}^{*}$ with one-dimensional kernel $\mathbb{C}\left(\varepsilon_{0}+\cdots+\varepsilon_{n}\right)$. In this case $Q \subset \mathfrak{h}^{*}$ is the root lattice. By $W$ we denote the Weyl group of $\mathfrak{g}$. Denote by $Z:=Z(U)$ the center of $U$ and let $Z^{\prime}:=\operatorname{Hom}(Z, \mathbb{C})$ be the set of all central characters (here Hom stands for homomorphisms of unital $\mathbb{C}$-algebras). By $\chi_{\lambda} \in Z^{\prime}$ we denote the central character of the irreducible highest weight module with highest weight $\lambda$. Recall that $\chi_{\lambda}=\chi_{\mu}$ iff $\lambda+\rho=w(\mu+\rho)$ for some element $w$ of the Weyl group $W$, where, as usual, $\rho$ denotes the half-sum of positive roots. We say that $\chi=\chi_{\lambda}$ is regular if the stabilizer of $\lambda+\rho$ in $W$ is trivial (otherwise $\chi$ is called singular), and that $\chi=\chi_{\lambda}$ is integral if $\lambda$ is in the weight lattice (i.e. in the lattice spanned by the fundamental weights $\left.\gamma\left(\varepsilon_{0}+\ldots+\varepsilon_{i-1}\right), i=1, \ldots, n\right)$. We say that two weights $\lambda$ and $\nu \in \lambda+\Lambda$ are in the same Weyl chamber if for any positive root $\alpha$ such that $(\lambda, \alpha) \in \mathbb{Z},(\lambda, \alpha) \in \mathbb{Z}_{\geq 0}$ if and only if $(\mu, \alpha) \in \mathbb{Z}_{\geq 0}$. Finally, recall that $\lambda$ is dominant integral if $(\lambda, \alpha) \in \mathbb{Z}_{\geq 0}$ for all positive roots $\alpha$.

One should note that every generalized bounded module has finite Jordan-Hölder series (see Lemma 3.3 in [19]). Since the center $Z$ of $U$ preserves weight spaces, it acts locally finitely on the generalized bounded modules. For every central character $\chi \in Z^{\prime}$ let $\mathcal{G B}^{\chi}$ (respectively, $\mathcal{B}^{\chi}, \mathcal{G C}^{\chi}, \mathcal{C}^{\chi}$ ) denote the category of all generalized bounded modules (respectively, bounded, generalized cuspidal, cuspidal) modules $M$ with generalized central character $\chi$, i.e. such that for some $n(M),(z-\chi(z))^{n(M)}=$ 0 on $M$ for all $z \in Z$. It is clear that every generalized bounded module $M$ is a direct sum of finitely many $M_{i} \in \mathcal{G B}^{\chi_{i}}$. Thus, one can write

$$
\mathcal{G B}=\bigoplus_{\substack{\chi \in Z^{\prime} \\ \bar{\mu} \in \mathfrak{h}^{*} / Q}} \mathcal{G} \mathcal{B}_{\bar{\mu}}^{\chi}, \mathcal{G C}=\bigoplus_{\substack{\chi \in Z^{\prime} \\ \bar{\mu} \in \mathfrak{h}^{*} / Q}} \mathcal{G} \mathcal{C}_{\bar{\mu}}^{\chi}, \mathcal{B}=\bigoplus_{\substack{\chi \in Z^{\prime} \\ \bar{\mu} \in \mathfrak{h}^{*} / Q}} \mathcal{B}_{\bar{\mu}}^{\chi}, \mathcal{C}=\bigoplus_{\substack{\chi \in Z^{\prime} \\ \bar{\mu} \in \mathfrak{h}^{*} / Q}} \mathcal{C}_{\bar{\mu}}^{\chi}
$$

where $\mathcal{G B}_{\bar{\mu}}^{\chi}=\mathcal{G B}^{\chi} \cap \mathcal{G B}_{\bar{\mu}}$, etc. Note that many of the direct summands above are trivial.

By $\chi_{\lambda}$ we denote the central character of the simple highest weight $\mathfrak{g}$-module with highest weight $\lambda$. For simplicity we put $\mathcal{G B}^{\lambda}:=\mathcal{G B}^{\chi_{\lambda}}, \mathcal{G B}_{\bar{\mu}}^{\lambda}:=\mathcal{G B}_{\bar{\mu}}^{\chi_{\lambda}}$, etc.

Let $\overline{\mathcal{B}}$ (respectively, $\overline{\mathcal{G B}}$ ) be the full subcategory of all weight modules (respectively, generalized weight modules) consisting of $\mathfrak{g}$-modules $M$ whose finitely generated submodules belong to $\mathcal{B}$ (respectively, $\mathcal{G} B$ ). It is not hard to see that every such $M$ is a direct $\operatorname{limit} \lim M_{i}$ for some directed system $\left\{M_{i} \mid i \in I\right\}$ such that each $M_{i} \in \mathcal{G B}$ (respectively, $\overrightarrow{M_{i}} \in \mathcal{B}$ ). It implies that the action of the center $Z$ of the universal enveloping algebra $U$ on $M$ is locally finite and we have decompositions

$$
\overline{\mathcal{B}}=\bigoplus_{\substack{\chi \in Z^{\prime} \\ \bar{\mu} \in \mathfrak{h}^{*} / Q}} \overline{\mathcal{B}}_{\bar{\mu}}^{\chi}, \overline{\mathcal{G B}}=\bigoplus_{\substack{\chi \in Z^{\prime} \\ \bar{\mu} \in \mathfrak{h}^{*} / Q}} \overline{\mathcal{G B}}_{\bar{\mu}}^{\chi}
$$

In a similar way we define $\overline{\mathcal{C}}$ and $\overline{\mathcal{G C}}$ and obtain their block decompositions. Finally, we set $\overline{\mathcal{G B}}_{\bar{\mu}}^{\lambda}:=\overline{\mathcal{G B}}_{\bar{\mu}}^{\chi_{\lambda}}, \overline{\mathcal{B}}_{\bar{\mu}}^{\lambda}:=\overline{\mathcal{B}}_{\bar{\mu}}^{\chi_{\lambda}}$, etc. For a detailed discussion of $\overline{\mathcal{C}}$ we refer the reader to $\S 5$ of $[18$. 


\section{Some Quivers AND AlgEbras RElated to HyPERCUBES}

In this section we define certain pointed algebras which describe blocks of categories which we study in the paper.

4.1. Definitions. Let $k \geq 1$ and $C(k)$ be the quiver of the skeleton of the $k$ dimensional cube: the vertices of the quiver are the same as those of the cube. Arrows of $C(k)$ correspond to the edges of the cube, each edge gives rise to two arrows in $C(k)$ with opposite orientation.

We define an equivalence relation on the paths of $C(k)$ as follows. Two paths with the same beginning and end and of the same length are equivalent. Let $B(k)$ be the quotient of $\mathbb{C}[C(k)]$ by the relations identifying all equivalent paths.

For instance, the quiver for $B(2)$ is

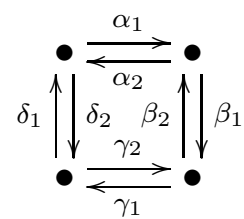

with relations $\beta_{1} \alpha_{1}=\gamma_{2} \delta_{2}, \alpha_{2} \alpha_{1}=\delta_{1} \delta_{2}, \ldots$ etc.

We define the algebras $B^{\prime}(k)$ for $k \geq 2$ (respectively, $B^{\prime \prime}(k)$ for $k \geq 3$ ) as the path algebras of $C(k)$ with one vertex (respectively, two opposite vertices) removed subject to the same relations described above. By $B^{\prime \prime}(2)$ we denote the path algebra of the quiver

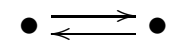

Note that $B^{\prime \prime}(2)=B(1)$ by definition.

Next we color the arrows of $C(k)$ by $k$ colors, in such a way, that two arrows have the same color if and only if the corresponding edges of the cube are parallel. As a result, to every path in $C(k)$ we associate a sequence of colors. We call two paths $p$ and $q$ equivalent if they have the same beginning and end and the sequence of colors of $p$ is obtained from that of $q$ by some permutation. Let $A(k)$ be the quotient of the path algebra $\mathbb{C}[C(k)]$ by the relations identifying all equivalent colored paths.

For $k \geq 2$ let $C^{\prime}(k)$ be obtained from $C(k)$ by removing one vertex from $C(k)$ and attaching a loop to each vertex adjacent to the removed one. In this way each loop at a vertex $v$ is a replacement of the path of length 2 from $v$ to the ghost (removed) vertex and back. We again associate to each path a sequence of colors with the convention that to the loop at $v$ we associate the sequence $(c, c)$, where $c$ is the color of the edge joining $v$ with the ghost vertex. Introduce the equivalence relation on the paths of $C^{\prime}(k)$ in the same manner as above and define $A^{\prime}(k)$ be the quotient of the path algebra $\mathbb{C}\left[C^{\prime}(k)\right]$ by the relations identifying all equivalent paths. 
For example $A^{\prime}(2)$ is the quotient of the path algebra of the quiver

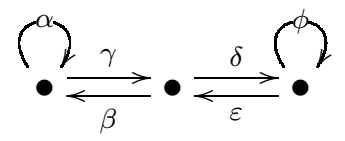

by the relations

$$
\alpha \beta \gamma=\beta \gamma \alpha, \gamma \beta \varepsilon \delta=\varepsilon \delta \gamma \beta, \delta \varepsilon \phi=\phi \delta \varepsilon .
$$

If $k \geq 3$ we consider also the quiver $C^{\prime \prime}(k)$ obtained from $C(k)$ by removing two opposite vertices and attaching loops to the vertices adjacent to the removed ones. We define the coloring of paths and construct the algebra $A^{\prime \prime}(k)$ like in the previous case.

For $k=2$ define $C^{\prime \prime}(2)$ to be the quiver

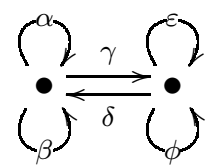

By $A^{\prime \prime}(2)$ we denote the quotient of path algebra of $C^{\prime \prime}(2)$ by the relations $\alpha \beta=$ $\beta \alpha=\delta \gamma, \varepsilon \phi=\phi \varepsilon=\gamma \delta$.

\subsection{Tameness.}

Proposition 4.1. (a) $B(k)$ is tame if and only if $k=1,2$.

(b) $B^{\prime}(k)$ is tame if and only if $k=2$.

(c) $B^{\prime \prime}(k)$ is tame if and only if $k=2$.

Proof. Observe that $B(1)$ is the path algebra of the quiver $A_{1}^{(1)}$, which is tame (see [11, 222). To prove the tameness of $B(2)$ we show that it is a skewed-gentle algebra which is tame by [16] (see also [6]). To prove that it is skewed-gentle, we consider the quiver of type $A_{1}^{(1)}$ with the relations $\alpha \beta=\beta \alpha=0$, where $\alpha$ and $\beta$ are two arrows of the quiver, and set both vertices to be special. Then the corresponding skewed-gentle algebra coincides with $B(2)$.

The wildness of $B^{\prime}(k)$ and $B^{\prime \prime}(\ell)$ for $k \geq 3$ and $\ell \geq 4$ follows from the fact that both algebras have a quotient algebra which is isomorphic to the path algebra of the quiver

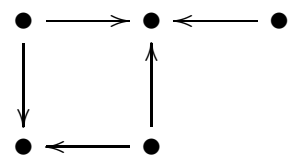

without relations. The tameness of $B^{\prime}(2)$ is a standard fact - see for example $§ V I I I .7 .9$ in [12]. 
It remains to show that $B^{\prime \prime}(3)$ is wild. The quiver for $B^{\prime \prime}(3)$ is

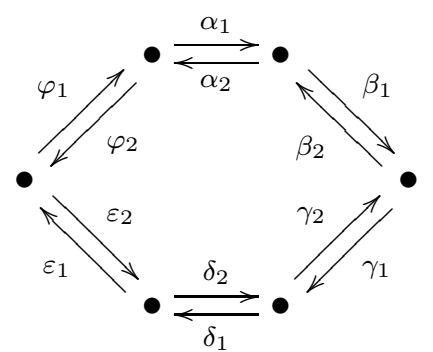

subject to the relations that every two paths with the same starting and end point and of same length are equal. We claim that the algebra $E=B^{\prime \prime}(3) / \operatorname{rad}^{3}\left(B^{\prime \prime}(3)\right)$ is wild. To show this we consider the universal cover for $E$, see [15], and notice that it has the following subquiver

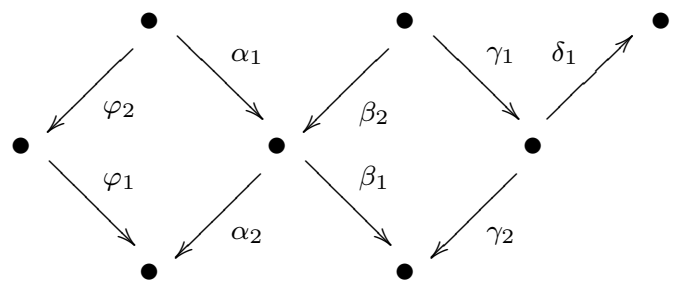

subject to the relations $\varphi_{1} \varphi_{2}=\alpha_{2} \alpha_{1}, \beta_{1} \beta_{2}=\gamma_{2} \gamma_{1}$. This quiver is wild, see for instance [23]. Hence $E$ and $B^{\prime \prime}(3)$ are wild.

Proposition 4.2. $A(k), A^{\prime}(k)$ and $A^{\prime \prime}(k)$ are wild for any $k \geq 2$, while $A(1)$ is tame.

Proof. First, we consider the case $k=2$. For $A^{\prime \prime}(2)$ we take its quotient by $\alpha=\gamma=$ $\varepsilon=0$, and for $A^{\prime}(2)$ we consider the quotient by $\gamma=\delta=\varepsilon=\sigma=0$. In both cases we obtain the path algebra of the quiver

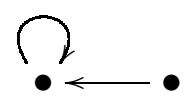

which is wild. For $A(2)$ in a similar way one constructs a quotient algebra isomorphic to the path algebra of the quiver $\bullet \rightleftarrows \bullet \longleftarrow \bullet$.

For $k>2, A(k), A^{\prime}(k)$ and $A^{\prime \prime}(k)$ always have a quotient algebra isomorphic to $A^{\prime}(2)$.

Finally we notice that the category of nilpotent representations of $A(1)$ is isomorphic to the category of $\mathbb{Z}_{2}$-graded nilpotent representations of $\mathbb{C}[\theta]$ with $\operatorname{deg}(\theta)=1$. Hence, this category is tame.

\subsection{Koszulity.}

Proposition 4.3. The algebras $A(k)$ and $B(k)$ for $k \geq 2$ are Koszul. 
Proof. Let us enumerate the vertices of the quiver $C_{k}$ by the elements of the group $G_{k} \simeq \mathbb{Z}_{2}^{k}$ in the natural way. By $\delta_{1}, \ldots, \delta_{k}$ we denote the basis of $G_{k}$ over $\mathbb{Z}_{2}$. We also consider the inner product $(\cdot, \cdot)$ on $G_{k}$ such that $\left(\delta_{i}, \delta_{j}\right)=\delta_{i j}$.

Define the $G_{k}$-grading of the polynomial algebra $\mathbb{C}\left[x_{1}, \ldots, x_{k}\right]$ by setting the degree of $x_{i}$ to equal $\delta_{i}$. In addition, we consider the standard $\mathbb{Z}$-grading of $\mathbb{C}\left[x_{1}, \ldots, x_{k}\right]$. Thus, $\mathbb{C}\left[x_{1}, \ldots, x_{k}\right]$ is now equipped with a $G_{k} \times \mathbb{Z}$-grading. It is not hard to see that the category of all $\mathbb{Z}$-graded $A(k)$-modules is equivalent to the category of all $G_{k} \times$ $\mathbb{Z}$-graded $\mathbb{C}\left[x_{1}, \ldots, x_{k}\right]$-modules. The Koszul resolution of the trivial $\mathbb{C}\left[x_{1}, \ldots, x_{k}\right]$ module can be equipped with a $G_{k}$-grading. Therefore $A(k)$ is Koszul.

To prove the Koszulity of $B(k)$, let us first renormalize the arrows of the quiver $C(k)$ in the following way. If the arrow $\theta$ joins the vertices $v$ and $v+\delta_{i}$, where $v \in G_{k}$, we multiply $\theta$ by $(-1)^{\left(v, \delta_{1}+\cdots+\delta_{i}\right)}$. Let $D(k)$ be the quadratic algebra with generators $\xi_{1}, \ldots, \xi_{k}$ and relations $\xi_{i} \xi_{j}+\xi_{j} \xi_{i}=0, \xi_{i}^{2}=\xi_{j}^{2}$ for all $i \neq j$. Note that $D(k)$ is the dual to the Koszul algebra $\mathbb{C}\left[x_{1}, \ldots, x_{k}\right] /\left(x_{1}^{2}+\cdots+x_{k}^{2}\right)$ and, hence, $D(k)$ is Koszul. Define a $G_{k}$-grading on $D(k)$ by setting the degree of $x_{i}$ to equal $\delta_{i}$. Then the category of all graded $B(k)$-modules is equivalent to the category of $G_{k} \times \mathbb{Z}$-graded $D(k)$-modules. Thus, the Koszulty of $B(k)$ follows by the argument analogous to the one in the previous case.

Finally let us note that $B^{\prime}(k)$ for $k \geq 3$ and $B^{\prime \prime}(k)$ for $k \geq 4$ are quadratic algebras. However, we do not know if they are Koszul.

\section{Generalized Weight modules over the Weyl Algebra}

The goal of this section is to study the structure of ${ }^{\mathrm{b}}(\mathcal{D}, \mathcal{H})_{\mu}-\bmod$. Note that since the simple modules in $(\mathcal{D}, \mathcal{H})$-mod are the same as those in ${ }^{\mathrm{wb}}(\mathcal{D}, \mathcal{H})$-mod, we can use the description of simples from [17]. We will show that $(\mathcal{D}, \mathcal{H})$-mod has enough injectives and explicitely construct an injective cogenerator $\mathcal{R}_{\mu}$ in $(\mathcal{D}, \mathcal{H})_{\mu}-\bmod$.

We use a slight modification of the classical result of Gabriel. For the proof of this version see [18].

Theorem 5.1. Let $\mathcal{R}_{\mu}$ be an injective cogenerator of $(\mathcal{D}, \mathcal{H})_{\mu}-\bmod$, and let $\mathcal{A}_{\mu}=$ $\operatorname{End}_{\mathcal{D}}\left(\mathcal{R}_{\mu}\right)$. The category $\mathcal{A}_{\mu}$-fmod of finite dimensional $\mathcal{A}_{\mu}$-modules is antiequivalent to the category ${ }^{\mathrm{b}}(\mathcal{D}, \mathcal{H})_{\mu}$-mod. The mutually inverse contravariant functors which establish this antiequivalence are $\operatorname{Hom}_{\mathcal{D}}\left(\cdot, \mathcal{R}_{\nu}\right)$ and $\operatorname{Hom}_{\mathcal{A}_{\mu}}\left(\cdot, \mathcal{R}_{\nu}\right)$.

5.1. The case of $\mathcal{D}(1)$. In this subsection we assume $\mathcal{D}=\mathcal{D}(1), t_{0}=t$ and $\partial_{0}=\partial$. For $\nu \in \mathbb{C}$ we set

$$
\mathcal{F}_{\nu}=t^{\nu} \mathbb{C}\left[t, t^{-1}\right]
$$

and consider $\mathcal{F}_{\nu}$ as a $\mathcal{D}$-module with the natural action of $\mathcal{D}$. It is easy to check that $\mathcal{F}_{\nu} \in{ }^{\mathrm{b}}(\mathcal{D}, \mathcal{H})_{\mu}-\bmod$ and $\mathcal{F}_{\nu}$ is simple iff $\nu \notin \mathbb{Z}$. By definition, $\mathcal{F}_{\nu} \simeq \mathcal{F}_{\mu}$ if and only if $\mu-\nu \in \mathbb{Z}$. So, if $\nu \in \mathbb{Z}$ we may assume $\nu=0$. It is an easy exercise to check that $\mathcal{F}_{0}$ has length 2 and one has the following non-split exact sequence

$$
0 \rightarrow \mathcal{F}_{0}^{+} \rightarrow \mathcal{F}_{0} \rightarrow \mathcal{F}_{0}^{-} \rightarrow 0
$$


where $\mathcal{F}_{0}^{+}=\mathbb{C}[t]$ and $\mathcal{F}_{0}^{-}$is a simple quotient. Moreover, if $\sigma$ denotes the automorphism of $\mathcal{D}$ defined by $\sigma(t)=\partial, \sigma(\partial)=-t$, then $\mathcal{F}_{0}^{-} \simeq\left(\mathcal{F}_{0}^{+}\right)^{\sigma}$.

As follows for instance from [17, any simple object in $(\mathcal{D}, \mathcal{H})$-mod is isomorphic to $\mathcal{F}_{\nu}$ for some non-integer $\nu, \mathcal{F}_{0}^{-}$or $\mathcal{F}_{0}^{+}$. In this subsection, we will verify that $(\mathcal{D}, \mathcal{H})$-mod has enough injectives.

Set $u=\log t$ and consider the $\mathcal{D}$-module

$$
\mathcal{F}_{\nu}^{\log }=\mathcal{F}_{\nu} \otimes \mathbb{C}[u]
$$

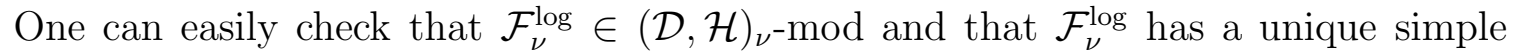
submodule isomorphic to $\mathcal{F}_{\nu}$ for $\nu \notin \mathbb{Z}$ and to $\mathcal{F}_{0}^{+}$for $\nu \in \mathbb{Z}$.

Proposition 5.2. The module $\mathcal{F}_{\nu}^{\log }$ is an injective module in the category $(\mathcal{D}, \mathcal{H})$ mod.

Proof. If $\nu \in \mathbb{Z}$ we assume $\nu=0$. Let $N=\mathcal{F}_{\nu}^{\log }$. For any $\mu \in \operatorname{supp} N$ the generalized weight subspace $N^{(\mu)}$ is isomorphic to the direct limit

$$
\underset{\lim }{\longrightarrow} \mathcal{H} /(t \partial-\mu)^{m}
$$

Hence $N^{(\mu)}$ is injective in the category of locally finite $\mathcal{H}$-modules.

For any $\mathcal{D}$-module $M$ let $\Gamma_{\mathcal{H}}(M)$ be the set of all finite $\mathcal{H}$-vectors. Note that $\Gamma_{\mathcal{H}}(M)$ is in fact $\mathcal{D}$-invariant and therefore

$$
\Gamma_{\mathcal{H}}: \mathcal{D}-\bmod \rightarrow(\mathcal{D}, \mathcal{H})-\bmod
$$

is a functor right adjoint to the embedding functor $(\mathcal{D}, \mathcal{H})-\bmod \rightarrow \mathcal{D}-\bmod$.

We claim now that

$$
I_{\nu}=\Gamma_{\mathcal{H}}\left(\operatorname{Hom}_{\mathcal{H}}\left(\mathcal{D}, N^{(\nu)}\right)\right)
$$

is injective in $(\mathcal{D}, \mathcal{H})$-mod. Indeed, for any $M \in(\mathcal{D}, \mathcal{H})$-mod

$$
\operatorname{Hom}_{\mathcal{D}}\left(M, I_{\nu}\right)=\operatorname{Hom}_{\mathcal{D}}\left(M, \operatorname{Hom}_{\mathcal{H}}\left(\mathcal{D}, N^{(\nu)}\right)\right)=\operatorname{Hom}_{\mathcal{H}}\left(M, N^{(\nu)}\right),
$$

where the second equality follows from the Frobenius reciprocity. Thus, $\operatorname{Hom}_{\mathcal{D}}\left(\cdot, I_{\nu}\right) \simeq$ $\operatorname{Hom}_{\mathcal{H}}\left(\cdot, N^{(\nu)}\right)$ is an exact functor.

It remains to prove that $N$ is isomorphic to $I_{\nu}$. Consider the homomorphism $\varphi: N \rightarrow I_{\nu}$ induced by the projection $N \rightarrow N^{(\nu)}$ via Frobenius reciprocity. Then $\varphi$ is injective since it is not zero on the unique simple submodule of $N$. To prove the surjectivity of $\varphi$ note that any generalized weight subspace $I_{\nu}^{(\mu)}$ is isomorphic to $N^{(\mu)}$ and any non-zero $\mathcal{H}$-linear map $I_{\nu}^{(\mu)} \rightarrow N^{(\mu)}$ is surjective.

It is clear from above that we have constructed injective hulls of all up to isomorphism simple objects in $(\mathcal{D}, \mathcal{H})$-mod except of $\mathcal{F}_{0}^{-}$. To construct an indecomposable injective hull of $\mathcal{F}_{0}^{-}$we use the twist by $\sigma$. Indeed, $\left(\mathcal{F}_{0}^{\log }\right)^{\sigma}$ is injective with unique simple submodule $\left(\mathcal{F}_{0}^{+}\right)^{\sigma} \simeq \mathcal{F}_{0}^{-}$.

Thus, we have constructed an injective cogenerator $\mathcal{R}_{\nu}$ for every block $(\mathcal{D}, \mathcal{H})_{\nu}-$ mod. Namely, if $\nu \notin \mathbb{Z}$ we have $\mathcal{R}_{\nu}=\mathcal{F}_{\nu}^{\log }$, and if $\nu=0$ we have $\mathcal{R}_{0}=\mathcal{F}_{0}^{\log } \oplus\left(\mathcal{F}_{0}^{\log }\right)^{\sigma}$. 
Lemma 5.3. $\operatorname{End}_{\mathcal{D}}\left(\mathcal{F}_{\nu}^{\text {log }}\right) \simeq \mathbb{C}[[z]]$.

Proof. First, we consider the embedding $j: C[[z]] \rightarrow \operatorname{End}_{\mathcal{D}}\left(\mathcal{F}_{\nu}^{\log }\right)$ defined by $z \mapsto \frac{\partial}{\partial u}$. It remains to prove that $j$ is surjective. Note that $\mathcal{F}_{\nu}^{\text {log }}$ is equipped with an increasing exhausting filtration

$$
0=F_{0} \subset F_{1} \subset F_{2} \subset \ldots,
$$

with $F_{1}=\mathcal{F}_{\nu}$ and $F_{k}=F_{k-1}+u F_{k-1}$. We have

$$
\operatorname{Hom}_{\mathcal{D}}\left(F_{k} / F_{k-1}, F_{1}\right) \simeq \mathbb{C} \text {. }
$$

Every $f \in \operatorname{End}_{\mathcal{D}}\left(\mathcal{F}_{\nu}^{\text {log }}\right)$ preserves the filtration and hence we have

$$
\operatorname{End}_{\mathcal{D}}\left(\mathcal{F}_{\nu}^{\log }\right)=\lim _{\longleftarrow} \operatorname{End}_{\mathcal{D}}\left(F_{k}\right) .
$$

It follows easily from (5.1) that $\operatorname{End}_{\mathcal{D}}\left(F_{k}\right) \simeq \mathbb{C}[z] /\left(z^{k+1}\right)$. This completes the proof.

Corollary 5.4. $\operatorname{End}_{\mathcal{D}}\left(\left(\mathcal{F}_{0}^{\log }\right)^{\sigma}\right) \simeq \mathbb{C}[[z]]$.

Lemma 5.5. There exists $\theta \in \operatorname{End}_{\mathcal{D}}\left(\mathcal{R}_{0}\right)$ such that $\theta\left(\mathcal{F}_{0}^{\log }\right)=\left(\mathcal{F}_{0}^{\log }\right)^{\sigma}, \theta\left(\left(\mathcal{F}_{0}^{\log }\right)^{\sigma}\right)=$ $\mathcal{F}_{0}^{\log }$ and $\theta^{2}=z$.

Proof. Recall that $\mathcal{F}_{0} / \mathcal{F}_{0}^{+} \simeq \mathcal{F}_{0}^{-}$, and this isomorphism extends to homomorphism $\theta^{+}: \mathcal{F}_{0}^{\log } \rightarrow\left(\mathcal{F}_{0}^{\log }\right)^{\sigma}$ with kernel $\mathcal{F}_{0}^{+}$. By twisting with $\sigma$ we construct $\theta^{-}:\left(\mathcal{F}_{0}^{\log }\right)^{\sigma} \rightarrow$ $\mathcal{F}_{0}^{\log }$ with kernel $\mathcal{F}_{0}^{-}$. By construction

$$
\operatorname{Ker}\left(\theta^{-} \circ \theta^{+}\right)=\mathcal{F}_{0}
$$

and

$$
\operatorname{Ker}\left(\theta^{+} \circ \theta^{-}\right)=\mathcal{F}_{0}^{\sigma}
$$

That implies $\theta^{-} \circ \theta^{+}=z f$ for some unit $f \in \mathbb{C}[z]$. By normalizing we can assume that $f=1$. Finally, set $\theta=\theta^{+}+\theta^{-}$.

Corollary 5.6. The algebra $\operatorname{End}_{\mathcal{D}}\left(\mathcal{R}_{0}\right)$ is generated by $\mathbb{C}[[\theta]]$ and the idempotents $e^{+}$and $e^{-}$representing projectors on $\mathcal{F}_{0}^{\log }$ and $\left(\mathcal{F}_{0}^{\log }\right)^{\sigma}$, respectively.

Theorem 5.7. Let $\nu \in \mathbb{C}$.

(a) If $\nu \notin \mathbb{Z}$, then ${ }^{\mathrm{b}}(\mathcal{D}, \mathcal{H})_{\nu}$-mod is equivalent to the category of finite-dimensional $\mathbb{C}[z]$-modules with nilpotent action of $z$.

(b) If $\nu \in \mathbb{Z}$, then ${ }^{\mathrm{b}}(\mathcal{D}, \mathcal{H})_{\nu}$-mod is equivalent to the category of finite-dimensional $\mathbb{Z}_{2}$-graded $\mathbb{C}[\theta]$-modules with nilpotent action of $\theta$, where the $\mathbb{Z}_{2}$-grading on $\mathbb{C}[\theta]$ is defined by $\operatorname{deg} \theta=1$.

Proof. The theorem is an easy consequence of Theorem 5.1, Lemma 5.3, and Corollary 5.6.

Indeed, in the first case $\mathcal{A}_{\nu}=\mathbb{C}[[z]]$ and the category $\mathcal{A}_{\nu}$-fmod is equivalent to the category finite-dimensional $\mathbb{C}[z]$-modules with nilpotent action of $z$. Moreover, 
the latter category clearly has a nice duality functor. Hence, we can change the antiequivalence by an equivalence.

In the second case $\mathcal{A}_{\nu}$ is generated by $\mathbb{C}[[\theta]]$ and two idempotents. Clearly $\theta$ acts nilpotently on all finite-dimensional $\mathbb{C}[[\theta]]$-modules. The idempotents correspond to projectors on the homogeneous parts of the $\mathbb{Z}_{2}$-grading.

Observe that, by the above theorem, ${ }^{\mathrm{b}}(\mathcal{D}, \mathcal{H})_{\nu}$-mod is tame. If $\nu \notin \mathbb{Z}$, all indecomposable representations in this category are parametrized by their dimension that can be any positive integer. If $\nu \in \mathbb{Z}$, the indecomposable representations are enumerated by their superdimension which can take values $(n+1, n),(n, n+1)$ and $(n, n)$. In the latter case there are two up to isomorphism indecomposable representations with the same superdimension, and one is obtained from the other by changing the parity.

5.2. The case of $\mathcal{D}(n+1)$ for arbitrary $n$. We assume now $\mathcal{D}=\mathcal{D}(n+1)$ and repeatedly use the fact that $\mathcal{D}(n+1)$ is the tensor product of $n+1$ copies of $\mathcal{D}(1)$. We set $\mathcal{F}_{\nu}^{\log }=\mathcal{F}_{\nu_{0}}^{\log } \otimes \ldots \otimes \mathcal{F}_{\nu_{n}}^{\log }$ and $\mathcal{F}_{\nu}=\mathcal{F}_{\nu_{0}} \otimes \ldots \otimes \mathcal{F}_{\nu_{n}}$.

It follows from [17] that for $\nu=\left(\nu_{0}, \ldots, \nu_{n}\right)$ every simple module in $(\mathcal{D}, \mathcal{H})_{\nu^{-}}$ mod is the tensor product $S_{0} \otimes S_{1} \otimes \cdots \otimes S_{n}$ where each $S_{i}$ is a simple module in $(\mathcal{D}(1), \mathcal{H}(1))_{\nu_{i}}-\bmod$.

Let $\operatorname{Int}(\nu)$ be the set of all $i$ such that $\nu_{i} \in \mathbb{Z}$ and $\mathcal{P}(\nu)$ be the power set of $\operatorname{Int}(\nu)$. For every $J \in \mathcal{P}(\nu)$ set

$$
\mathcal{S}_{\nu}(J):=S_{0} \otimes S_{1} \otimes \cdots \otimes S_{n},
$$

where $S_{i}=\mathcal{F}_{\nu_{i}}$ if $i \notin \operatorname{Int}(\nu), S_{i}=\mathcal{F}_{0}^{+}$if $i \in \operatorname{Int}(\nu) \backslash J$ and $S_{i}=\mathcal{F}_{0}^{-}$if $i \in J$.

Lemma 5.8. Any simple object in $(\mathcal{D}, \mathcal{H})_{\nu}$-mod is isomorphic to $\mathcal{S}_{\nu}(J)$ for $J \in \mathcal{P}(\nu)$. Moreover, $\mathcal{S}_{\nu}(J) \simeq \mathcal{S}_{\nu}(I)$ if and only if $I=J$. If by $\sigma_{i}$ we denote the automorphsim of $\mathcal{D}$ induced by $\sigma$ on the $i$-th copy of $\mathcal{D}(1)$ and set $\sigma_{J}=\prod_{i \in J} \sigma_{i}$, then $\mathcal{S}_{\nu}(J)=$ $\left(\mathcal{S}_{\nu}(\emptyset)\right)^{\sigma_{J}}$.

Proof. The lemma follows from [17].

Similarly as above, for any $J \in \mathcal{P}(\nu)$ set

$$
\mathcal{I}_{\nu}(J):=I_{0} \otimes I_{1} \otimes \cdots \otimes I_{n}
$$

where $I_{j}=\mathcal{F}_{\nu_{j}}^{\log }$ if $j \notin \operatorname{Int}(\nu), I_{j}=\mathcal{F}_{0}^{\log }$ if $j \in \operatorname{Int}(\nu) \backslash J$ and $I_{j}=\left(\mathcal{F}_{0}^{\log }\right)^{\sigma}$ if $j \in J$. Observe that

$$
\mathcal{I}_{\nu}(\emptyset)=\prod_{i=0}^{n} t_{i}^{\nu_{i}} \mathbb{C}\left[t_{0}^{ \pm 1}, \ldots, t_{n}^{ \pm 1}, u_{0}, \ldots, u_{n}\right]
$$

with $u_{i}=\log \left(t_{i}\right)$. We also set $\mathcal{F}_{\nu}^{\log }(J)=\left(\mathcal{F}_{\nu}^{\log }\right)^{\sigma_{J}}$ and $\mathcal{F}_{\nu}(J)=\left(\mathcal{F}_{\nu}\right)^{\sigma_{J}}$

Lemma 5.9. (a) For any $J \in \mathcal{P}(\nu), \mathcal{I}_{\nu}(J)$ is the indecomposable injective hull of $\mathcal{S}_{\nu}(J)$ in the category $(\mathcal{D}, \mathcal{H})$-mod.

(b) $\mathcal{R}_{\nu}=\bigoplus_{J \in \mathcal{P}(\nu)} \mathcal{I}_{\nu}(J)$ is an injective cogenerator in $(\mathcal{D}, \mathcal{H})_{\nu}$-mod. 
Proof. (a) The proof in the case $J=\emptyset$ is the same as in the case $n=1$, see Proposition 5.2. For $J \neq \emptyset$, we use the twist by $\sigma_{J}$.

Part (b) follows from (a).

Let $\mathcal{A}_{\nu}=\operatorname{End}_{\mathcal{D}}\left(\mathcal{R}_{\nu}\right)$. Recall that $\mathcal{R}_{\nu}=\mathcal{R}_{\nu_{0}} \otimes \cdots \otimes \mathcal{R}_{\nu_{n}}$. Define a $\mathbb{Z}^{n+1}$-filtration on $\mathcal{R}_{\nu}$ by

$$
F_{m_{0}, \ldots, m_{n}}\left(\mathcal{R}_{\nu}\right)=F_{m_{0}}\left(\mathcal{R}_{\nu_{0}}\right) \otimes \cdots \otimes F_{m_{n}}\left(\mathcal{R}_{\nu_{n}}\right) .
$$

Again, it is not hard to see that this filtration is preserved by any $f \in A_{\nu}$. For each $\mathbf{m} \in \mathbb{Z}^{n+1}$ set

$$
A_{\nu}^{\mathbf{m}}=\operatorname{End}_{\mathcal{D}}\left(F_{\mathbf{m}}\left(\mathcal{R}_{\nu}\right)\right)
$$

Then

$$
\mathcal{A}_{\nu}=\lim _{\longleftarrow} \mathcal{A}_{\nu}^{\mathbf{m}}
$$

and

$$
\mathcal{A}_{\nu}^{\mathbf{m}}=\operatorname{End}_{\mathcal{D}(1)}\left(F_{m_{0}}\left(\mathcal{R}_{\nu_{0}}\right)\right) \otimes \cdots \otimes \operatorname{End}_{\mathcal{D}(1)}\left(F_{m_{n}}\left(\mathcal{R}_{\nu_{n}}\right)\right)
$$

For any $i \notin \operatorname{Int}(\nu)$ let $z_{i} \in \mathcal{A}_{\nu}$ be the endomorphism induced by $z$ on $\mathcal{R}_{\nu_{i}}$. If $i \in \operatorname{Int}(\nu)$ let $\theta_{i}, e_{i}^{+}, e_{i}^{-}$be induced by the corresponding endomorphisms of $\mathcal{R}_{\nu_{i}}$.

The proof of the following is straightforward.

Lemma 5.10. $\mathcal{A}_{\nu}$ is generated by $\mathbb{C}\left[\left[z_{i}, \theta_{j}\right]\right]_{i \notin \operatorname{Int}(\nu), j \in \operatorname{Int}(\nu)}$ and $e_{i}^{ \pm}$for all $i \in \operatorname{Int}(\nu)$.

Now applying Theorem 5.1 leads to the following result

Proposition 5.11. Assume that $|\operatorname{Int}(\nu)|=k$, and let

$$
G_{k}=\mathbb{Z}_{2}^{k}=\bigoplus_{i=1}^{k} \mathbb{Z}_{2} \delta_{i}
$$

Consider the $G_{k}$-grading on $\mathbb{C}\left[\theta_{1}, \ldots, \theta_{k}, z_{1}, \ldots, z_{n+1-k}\right]$ defined by $\operatorname{deg}\left(z_{i}\right)=0$ and $\operatorname{deg}\left(\theta_{j}\right)=\delta_{j}$. Then ${ }^{\mathrm{b}}(\mathcal{D}, \mathcal{H})_{\nu}$-mod is equivalent to the category of finite-dimensional $G_{k}$-graded $\mathbb{C}\left[\theta_{1}, \ldots, \theta_{k}, z_{1}, \ldots, z_{n+1-k}\right]$-modules with nilpotent action of all $z_{i}$ and $\theta_{j}$.

Note that for ${ }^{\mathrm{b}}(\mathcal{D}, \mathcal{H})_{\nu}$-mod is wild for $n>0$.

5.3. The category ${ }_{\mathrm{s}}^{\mathrm{b}}(\mathcal{D}, \mathcal{H})$-mod. Recall the definition of ${ }_{\mathrm{s}}^{\mathrm{b}}(\mathcal{D}, \mathcal{H})$-mod in Section 3.3 .

Define the left exact functor $S_{\mathcal{H}^{\prime}}:(\mathcal{D}, \mathcal{H})-\bmod \rightarrow{ }_{\mathrm{s}}(\mathcal{D}, \mathcal{H})$-mod to be the one that maps $M$ to its submodule consisting of all $\mathcal{H}^{\prime}$-eigenvectors. By general nonsense arguments, $S_{\mathcal{H}^{\prime}}$ maps injectives to injectives and blocks to blocks. Therefore, $S_{\mathcal{H}^{\prime}}\left(\mathcal{R}_{\nu}\right)$ is an injective cogenerator in the block ${ }_{\mathrm{s}}(\mathcal{D}, \mathcal{H})_{\nu}$-mod. It is not hard to see that

$$
S_{\mathcal{H}^{\prime}}\left(\mathcal{R}_{\nu}\right)=\mathcal{F}_{\nu} \otimes \mathbb{C}[u]
$$

where $u=\log \left(t_{0} t_{1} \cdots t_{n}\right)$. If we set $\mathcal{B}_{\nu}=\operatorname{End}\left(S_{\mathcal{H}^{\prime}}\left(\mathcal{R}_{\nu}\right)\right)$ then clearly $\mathcal{B}_{\nu}$ is the quotient of $\mathcal{A}_{\nu}$ by the ideal generated by $\frac{\partial}{\partial u_{i}}-\frac{\partial}{\partial u_{i}}$ for all $i \neq j$. This implies the following. 
Lemma 5.12. For any $\nu$, the ring $\mathcal{B}_{\nu}$ is the $\mathbb{C}[[z]]$-algebra generated by $\theta_{i}$ and the idempotents $e_{i}^{ \pm}$for all $i \in \operatorname{Int}(\nu)$, subject to the relations $\theta_{i}^{2}=z$.

Using Theorem 5.1 again, we obtain the following result.

Proposition 5.13. Assume that $|\operatorname{Int}(\nu)|=k>0$. Consider the $G_{k}$-grading on $\mathcal{B}(k)=\mathbb{C}\left[\theta_{1}, \ldots, \theta_{k}\right] /\left(\theta_{i}^{2}-\theta_{j}^{2}\right)$ defined by $\operatorname{deg}\left(\theta_{i}\right)=\delta_{i}$. Then ${ }_{\mathrm{s}}^{\mathrm{b}}(\mathcal{D}, \mathcal{H})_{\nu}$-mod is equivalent to the category of finite-dimensional $G_{k}$-graded $\mathcal{B}(k)$-modules with nilpotent action of all $\theta_{i}$. If $k=0$, then ${ }_{\mathrm{s}}^{\mathrm{b}}(\mathcal{D}, \mathcal{H})_{\nu}$-mod is equivalent to the category of finitedimensional $\mathbb{C}[z]$-modules with nilpotent action of $z$.

Corollary 5.14. The category ${ }_{\mathrm{s}}^{\mathrm{b}}(\mathcal{D}, \mathcal{H})_{\nu}$-mod is tame if and only if $|\operatorname{Int}(\nu)|=0,1$.

\section{6. $\mathcal{D}^{E}$ AND TWISTED DIFFERENTIAL OPERATORS ON $\mathbb{P}^{n}$}

In this section we assume that $n \geq 1$.

6.1. Connection between weight $\mathcal{D}$-modules and weight $\mathcal{D}^{E}$-modules. For any $M \in(\mathcal{D}, \mathcal{H})$-mod, let

$$
\Gamma_{a}(M)=\bigcup_{l>0} \operatorname{Ker}(E-a)^{l}
$$

It is not hard to see that $\Gamma_{a}$ is an exact functor from the category $(\mathcal{D}, \mathcal{H})$-mod to the category $\left(\mathcal{D}^{E}, \mathcal{H}\right)^{a}$-mod. The induction functor

$$
\Phi(X)=\mathcal{D} \otimes_{\mathcal{D}^{E}} X
$$

is its left adjoint.

Lemma 6.1. (a) $\Gamma_{a} \circ \Phi$ is isomorphic to the identity functor.

(b) If $S$ is simple in $(\mathcal{D}, \mathcal{H})$-mod, then $\Gamma_{a}(S)$ is either simple or zero.

(c) Let $S_{1}$ and $S_{2}$ be non-isomorphic simple modules in $(\mathcal{D}, \mathcal{H})$-mod and $\Gamma_{a}\left(S_{i}\right) \neq 0$ for $i=1,2$. Then $\Gamma_{a}\left(S_{1}\right)$ and $\Gamma_{a}\left(S_{2}\right)$ are not isomorphic.

(d) Any simple $M$ in $\left(\mathcal{D}^{E}, \mathcal{H}\right)^{a}$-mod is isomorphic to $\Gamma_{a}(S)$ for some simple $S$ in $(\mathcal{D}, \mathcal{H})$-mod.

(e) If $I$ is the indecomposable injective hull of a simple $S$ in $(\mathcal{D}, \mathcal{H})$-mod and $\Gamma_{a}(S) \neq 0$, then $\Gamma_{a}(I)$ is injective in $\left(\mathcal{D}^{E}, \mathcal{H}\right)^{a}$-mod.

Proof. (a) Use

$$
\Phi(X) \simeq \bigoplus_{m \in \mathbb{Z}} \mathcal{D}^{m} \otimes_{\mathcal{D}^{E}} X
$$

and

$$
\mathcal{D}^{m} \otimes_{\mathcal{D}^{E}} X=\bigcup_{l>0} \operatorname{Ker}(E-a-m)^{l} .
$$

Since $\mathcal{D}^{0}=\mathcal{D}^{E}$, we have

$$
\Gamma_{a}(X)=\mathcal{D}^{0} \otimes_{\mathcal{D}^{E}} X \simeq X
$$


(b) Note that both $S$ and $\Gamma_{a}(S)$ are multiplicity free weight modules. If $N$ is a proper non-zero submodule in $\Gamma_{a}(S)$, then $\operatorname{supp} N$ is a proper non-empty subset in $\operatorname{supp} \Gamma_{a}(S)$. Let $S^{\prime}$ be the submodule in $S$ generated by $N$. Then $\operatorname{supp} S^{\prime}$ is a proper non-empty subset in $\operatorname{supp} S$. That contradicts to the simplicity of $S$.

(c) The statement follows from the fact that $S_{1}$ and $S_{2}$ have disjoint supports.

(d) By (a), we have $M \simeq \Gamma_{a}(\Phi(M))$. Hence $M \simeq \Gamma_{a}(S)$ for some simple subquotient $S$ of $\Phi(M)$.

(e) Let $\varphi: X \rightarrow Y$ be an injective homomorphism of modules in $\left(\mathcal{D}^{E}, \mathcal{H}\right)^{a}$-mod, and $\eta: X \rightarrow \Gamma_{a}(I)$ be some homomorphism. Let $Z$ be the kernel of $\Phi(\varphi): \Phi(X) \rightarrow \Phi(Y)$, and $\eta^{\prime}: \Phi(X) \rightarrow I$ be the homomorphism induced by $\eta$. So we have $\Gamma_{a}\left(\eta^{\prime}\right)=\eta$. Note that by (a) we have $\Gamma_{a}(Z)=0$. We claim that $\eta^{\prime}(Z)=0$. Indeed, otherwise $\eta^{\prime}(Z)$ contains $S$, hence $Z$ has a subquotient isomorphic to $S$. But $\Gamma_{a}(S) \neq 0$. Then by the injectivity of $I$, there exists a homomorphism $\alpha: \Phi(Y) \rightarrow I$ such that $\eta^{\prime}=\alpha \circ \Phi(\varphi)$. After applying $\Gamma_{a}$ to the latter identity we obtain $\eta=\beta \circ \varphi$ with $\beta=\Gamma_{a}(\alpha)$.

Note that if $\nu \in \mathbb{C}^{n+1}$, then the restriction of $\Gamma_{a}$ to $(\mathcal{D}, \mathcal{H})_{\nu}$-mod is not identically zero if and only if $|\nu|-a \in \mathbb{Z}$.

Lemma 6.2. Let $\nu \notin \mathbb{Z}^{n+1}$ and $a-|\nu| \in \mathbb{Z}$. Then for any simple $S$ in $(\mathcal{D}, \mathcal{H})_{\nu}$-mod $\Gamma_{a}(S) \neq 0$.

Proof. We just have to check that the intersection of $\operatorname{supp} S$ with the hyperplane $|\mu|=a$ is not empty. This is an immediate consequence of

$$
\operatorname{supp}\left(\mathcal{S}_{\nu}(J)\right)=\sum_{i \in J} \mathbb{Z}_{<0} \varepsilon_{i}+\sum_{i \in \operatorname{Int}(\nu) \backslash J} \mathbb{Z}_{\geq 0} \varepsilon_{i}+\sum_{i \notin \operatorname{Int}(\nu)}\left(\nu_{i}+\mathbb{Z}\right) \varepsilon_{i}
$$

Corollary 6.3. Let $\nu \notin \mathbb{Z}^{n+1}$ and $a-|\nu| \in \mathbb{Z}$. Then $(\mathcal{D}, \mathcal{H})_{\nu}-\bmod$ and $\left(\mathcal{D}^{E}, \mathcal{H}\right)_{\nu}^{a}$-mod are equivalent.

Proof. From the previous lemma we have that $\Phi$ and $\Gamma_{a}$ are inverse.

6.2. On the structure of $\left(\mathcal{D}^{E}, \mathcal{H}\right)_{0}^{a}$-mod. We now consider the case $\nu \in \mathbb{Z}^{n+1}$. Without loss of generality we may assume $\nu=0$.

Lemma 6.4. Let $a \in \mathbb{Z}$.

(a) Let $-n-1<a<0$ and $\Gamma_{a}\left(\mathcal{S}_{0}(J)\right)=0$ if and only if $J=\emptyset$ or $J=\{0, \ldots, n+1\}$.

(b) If $a \geq 0$ and $\Gamma_{a}\left(\mathcal{S}_{0}(J)\right)=0$ if and only if $J=\{0, \ldots, n+1\}$.

(c) If $a \leq-n-1$ and $\Gamma_{a}\left(\mathcal{S}_{0}(J)\right)=0$ if and only if $J=\emptyset$.

Proof. Like the proof of Lemma 6.2, the statements follow by direct inspection using (6.1).

Consider the automorphism $\tau=\prod_{i=0}^{n} \sigma_{i}$ of $\mathcal{D}$. It is clear that $\tau(E)=-E-n-1$. Hence $\tau\left(\mathcal{D}^{E}\right)=\mathcal{D}^{E}$ and twist by $\tau$ induces an equivalence between $\left(\mathcal{D}^{E}, \mathcal{H}\right)_{a}$-mod

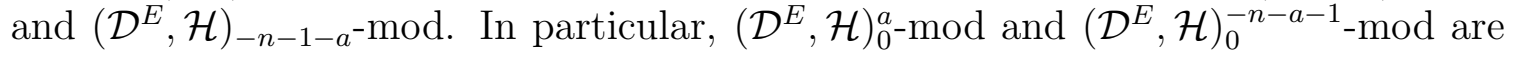


equivalent. Thus, without loss of generality, we can restrict our attention to the cases (a) and (b) of the last lemma.

If $a \geq 0$, then all up to isomorphism simple modules of $\left(\mathcal{D}^{E}, \mathcal{H}\right)_{0}^{a}$-mod are of the form $\mathcal{S}_{0}^{a}(J)=\Gamma_{a}\left(\mathcal{S}_{0}(J)\right)$, where $J$ runs through all proper subsets of $\{0, \ldots, n\}$. If $-n-1<a<0$, then all up to isomorphism simple modules of $\left(\mathcal{D}^{E}, \mathcal{H}\right)_{0}^{a}$-mod are of the form $\mathcal{S}_{0}^{a}(J)=\Gamma_{a}\left(\mathcal{S}_{0}(J)\right)$, where $J$ runs through all proper non-empty subsets of $\{0, \ldots, n\}$. In what follows we denote by $\mathcal{P}_{a}$ the collection of all proper subsets of $\{0, \ldots, n\}$ for $a \geq 0$, and that of all proper non-empty subsets of $\{0, \ldots, n\}$ for $-n-1<a<0$.

Set

$$
\mathcal{R}_{0}^{a}=\bigoplus_{J \in \mathcal{P}_{a}} \Gamma_{a}\left(\mathcal{I}_{0}(J)\right)
$$

Lemma 6.1 implies the following.

Corollary 6.5. $\mathcal{R}_{0}^{a}$ is an injective cogenerator in $\left(\mathcal{D}^{E}, \mathcal{H}\right)_{0}^{a}$-mod.

In view of Theorem 5.1, it is useful to study the algebra $\operatorname{End}_{\mathcal{D}^{E}}\left(\mathcal{R}_{0}^{a}\right)$. Let $\pi^{a} \in \mathcal{A}_{0}$ denote the projector onto $\bigoplus_{J \in \mathcal{P}_{a}} \mathcal{I}_{0}(J)$.

Lemma 6.6. $\operatorname{End}_{\mathcal{D}^{E}}\left(\mathcal{R}_{0}^{a}\right)$ is isomorphic to $\pi^{a} \mathcal{A}_{0} \pi^{a}$.

Proof. Observe that

$$
\pi^{a} \mathcal{A}_{0} \pi^{a}=\operatorname{End}_{\mathcal{D}}\left(\bigoplus_{J \in \mathcal{P}_{a}} \mathcal{I}_{0}(J)\right)
$$

We define a homomorphism $\gamma_{a}: \pi^{a} \mathcal{A}_{0} \pi^{a} \rightarrow \operatorname{End}_{\mathcal{D}^{E}}\left(\mathcal{R}_{0}^{a}\right)$ by setting $\gamma_{a}(g)=\Gamma_{a}(g)$. The injectivity of $\gamma_{a}$ follows immediately by definition. To prove the surjectivity consider $f \in \operatorname{End}_{\mathcal{D}^{E}}\left(\mathcal{R}_{0}^{a}\right)$. Note that $\Gamma_{a}$ has a right adjoint functor $\Phi^{\prime}$ which has all properties analogous to the left adjoint functor $\Phi$. It can be defined by

$$
\Phi^{\prime}(M)=\Gamma_{\mathcal{H}}\left(\operatorname{Hom}_{\mathcal{D}^{E}}(\mathcal{D}, M)\right) .
$$

By general nonsense arguments, $\Phi^{\prime}$ maps an injective module to an injective module and therefore

$$
\Phi^{\prime}\left(\mathcal{R}_{0}^{a}\right)=\bigoplus_{J \in \mathcal{P}_{a}} \mathcal{I}_{0}(J) .
$$

Then we have $f=\gamma_{a}\left(\Phi^{\prime}(f)\right)$.

In order to give a combinatorial description of ${ }^{\mathrm{b}}\left(\mathcal{D}^{E}, \mathcal{H}\right)_{0}^{a}$-mod we study certain quivers. By Proposition [5.11, $(\mathcal{D}, \mathcal{H})_{0}$-mod is equivalent to the category of finitedimensional nilpotent representations of $A(n+1)$, where by "nilpotent" we mean that every sufficiently long path acts trivially.

The following statement is now a consequence of Lemma 6.6.

Proposition 6.7. (a) If $a \geq 0$, then ${ }^{\mathrm{b}}\left(\mathcal{D}^{E}, \mathcal{H}\right)_{0}^{a}$-mod is equivalent to the category of nilpotent representations of $A^{\prime}(n+1)$. 
(b) If $-n-1<a<0$, then ${ }^{\mathrm{b}}\left(\mathcal{D}^{E}, \mathcal{H}\right)_{0}^{a}$-mod is equivalent to the category of nilpotent representations of $A^{\prime \prime}(n+1)$.

Corollary 6.8. For any $n \geq 1$, the category ${ }^{\mathrm{b}}\left(\mathcal{D}^{E}, \mathcal{H}\right)_{0}^{a}$-mod is wild.

6.3. On the structure of ${ }_{\mathrm{s}}\left(\mathcal{D}^{E}, \mathcal{H}\right)_{0}^{a}$-mod. Recall the definition of ${ }_{\mathrm{s}}\left(\mathcal{D}^{E}, \mathcal{H}\right)$ in Section 3.3. We can use the functor $\Gamma_{a}$ in the same way as in Section 6.1.

Proposition 6.9. Let $\nu \notin \mathbb{Z}^{n+1}$ and $a-|\nu| \in \mathbb{Z}$. Then ${ }_{\mathrm{s}}(\mathcal{D}, \mathcal{H})_{\nu^{-}} \bmod \operatorname{and}_{\mathrm{s}}\left(\mathcal{D}^{E}, \mathcal{H}\right)_{\nu^{-}}^{a}$ mod are equivalent.

Proof. The statement follows immediately from the analogue of Lemma 6.1.

Now we concentrate on the case $\nu \in \mathbb{Z}^{n+1}$ and, as before, assume that $\nu=0$. We apply the same arguments as in Section 6.1. We denote the idempotent of $\mathcal{B}(n+1)$ again by $\pi^{a}$. Recall that by Proposition 5.13, ${ }_{\mathrm{s}}^{\mathrm{b}}\left(\mathcal{D}^{E}, \mathcal{H}\right)_{0}^{a}$-mod is equivalent to the category of nilpotent $\mathcal{B}(n+1)$-modules. With this in mind, we have the following.

Lemma 6.10. The category $\mathrm{s}_{\mathrm{s}}^{\mathrm{b}}\left(\mathcal{D}^{E}, \mathcal{H}\right)_{0}^{a}$-mod is equivalent to the category of nilpotent $\pi^{a} \mathcal{B}(n+1) \pi^{a}$-modules.

We now formulate the above result in terms of quivers.

Proposition 6.11. (a) If $a \geq 0$, then ${ }_{\mathrm{s}}^{\mathrm{b}}\left(\mathcal{D}^{E}, \mathcal{H}\right)_{0}^{a}$-mod is equivalent to the category of nilpotent representations of $B^{\prime}(n+1)$. Therefore, ${ }_{\mathrm{s}}^{\mathrm{b}}\left(\mathcal{D}^{E}, \mathcal{H}\right)_{0}^{a}$-mod is tame if and only if $n=1$.

(b) If $-n-1<a<0$, then ${ }_{\mathrm{s}}^{\mathrm{b}}\left(\mathcal{D}^{E}, \mathcal{H}\right)_{0}^{a}$-mod is equivalent to the category of nilpotent representations of $B^{\prime \prime}(n+1)$. Therefore, ${ }_{\mathrm{s}}^{\mathrm{b}}\left(\mathcal{D}^{E}, \mathcal{H}\right)_{0}^{a}$-mod is tame if and only if $n=1$.

\section{Twisted Localization}

7.1. Twisted localization in general setting. Retain the notation of 93.1 . Namely, $\mathcal{U}$ is a finitely generated associative unital algebra, and $\mathcal{H}=\mathbb{C}\left[h_{0}, \ldots, h_{n}\right]=S(\mathfrak{h})$ is a subalgebra of $\mathcal{U}$ such that $\operatorname{ad}(h)$ is semisimple on $\mathcal{U}$ for every $h \in \mathfrak{h}$. Let now $F=\left\{f_{1}, \ldots, f_{k}\right\}$ be a subset of commuting elements of $\mathcal{U}$ such that ad $\left(f_{i}\right)$ are locally nilpotent endomorphisms of $\mathcal{U}$. Let $\langle F\rangle$ be the multiplicative subset of $\mathcal{U}$ generated by $\left\{f_{1}, \ldots, f_{k}\right\}$, i.e. the $\langle F\rangle$ consists of the elements $f_{1}^{k_{1}} \ldots f_{k}^{n_{k}}$ for $n_{i} \in \mathbb{Z}_{\geq 0}$. By $D_{F} \mathcal{U}$ we denote the localization of $\mathcal{U}$ relative to $\langle F\rangle$. Note that $\langle F\rangle$ satisfy Ore's localizability condition due to the fact that $f_{i}$ are locally ad-nilpotent. For a $\mathcal{U}$-module $M$, by $D_{F} M=D_{F} \mathcal{U} \otimes_{\mathcal{U}} M$ we denote the localization of $M$ relative to $\langle F\rangle$. We will consider $D_{F} M$ both as a $\mathcal{U}$-module and a $D_{F} \mathcal{U}$-module. By $\theta_{F}: M \rightarrow D_{F} M$ we denote the localization map defined by $\theta_{F}(m)=1 \otimes m$. Then

$$
\operatorname{ann}_{M} F:=\{m \in M \mid s m=0 \text { for some } s \in F\}
$$

is a submodule of $M$ (often called, the torsion submodule with respect to $F$ ). Note that if $\operatorname{ann}_{M} F=0$, then $\theta_{F}$ is an injection. In the latter case, $M$ will be considered 
naturally as a submodule of $D_{F} M$. Note also that if $F=F_{1} \cup F_{2}$, then $D_{F_{1}} D_{F_{2}} M \simeq$ $D_{F_{2}} D_{F_{1}} M \simeq D_{F} M$.

It is well known that $D_{F}$ is a functor from the category of $\mathcal{U}$-modules to the category of $D_{F} \mathcal{U}$-modules. For any category $\mathcal{A}$ of $\mathcal{U}$-modules, by $\mathcal{A}_{F}$ we denote the category of $D_{F} \mathcal{U}$-modules that considered as $\mathcal{U}$-modules are in $\mathcal{A}$. Some useful properties of the localization functor $D_{F}$ are listed in the following lemma.

Lemma 7.1. (i) If $\varphi: M \rightarrow N$ is a homomorphism of $\mathcal{U}$-modules, then $D_{F}(\varphi) \theta_{F}=$ $\theta_{F} \varphi$.

(ii) $D_{F}$ is an exact functor.

(iii) (Universal property) If $N$ is a $D_{F} \mathcal{U}$-module and $\varphi: M \rightarrow N$ is a homomorphism of $\mathcal{U}$-modules, then there exists a unique homomorphism of $D_{F} \mathcal{U}$-modules $\bar{\varphi}: D_{F} M \rightarrow N$ such that $\bar{\varphi} \theta_{F}=\varphi$. If we identify $N$ with $D_{F} N$, then $\bar{\varphi}=D_{F}(\varphi)$.

(iv) Let $\mathcal{A}$ be any category of $U$-modules. If $I$ is an injective module in $\mathcal{A}_{F}$, then $I$ (considered as an $\mathcal{U}$-module) is injective in $\mathcal{A}$ as well.

Proof. Statements (i)-(iii) are standard (see for example [21]). For (iv), consider a morphism $\varphi: A \rightarrow I$ and a monomorphism $\psi: A \rightarrow B$ in $\mathcal{A}$. By (ii), $D_{F} \psi$ is a monomorphism, and since $I$ is injective in $\mathcal{A}_{F}$, there is a morphism $\gamma: D_{F} A \rightarrow I$ such that $\gamma D_{F}(\psi)=D_{F}(\varphi)$. But then $\alpha:=\gamma \theta_{F}$ is a morphism from $B$ to $I$ and

$$
\alpha \psi=\gamma \theta_{F} \psi=\gamma D_{F}(\psi) \theta_{F}=D_{F}(\varphi) \theta_{F}=\varphi .
$$

The second identity follows from (i), while the last one from (iii).

We now introduce the "generalized conjugation" in $D_{F} \mathcal{U}$ following $\S 4$ of [19]. For $\mathbf{x}=\left(x_{1}, \ldots, x_{k}\right) \in \mathbb{C}^{k}$ define the automorphism $\Theta_{F}^{\mathbf{x}}$ of $D_{F} \mathcal{U}$ in the following way. For $u \in D_{F} U$ set

$$
\Theta_{F}^{\mathbf{x}}(u):=\sum_{i_{1}, \ldots, i_{k} \geq 0}\left(\begin{array}{c}
x_{1} \\
i_{1}
\end{array}\right) \ldots\left(\begin{array}{c}
x_{k} \\
i_{k}
\end{array}\right) \operatorname{ad}\left(f_{1}\right)^{i_{1}} \ldots \operatorname{ad}\left(f_{k}\right)^{i_{k}}(u) f_{1}^{-i_{1}} \ldots f_{k}^{-i_{k}},
$$

where $\left(\begin{array}{l}x \\ i\end{array}\right):=x(x-1) \ldots(x-i+1) / i$ ! for $x \in \mathbb{C}$ and $i \in \mathbb{Z}_{\geq 0}$. Note that for $\mathbf{x} \in \mathbb{Z}^{k}$, we have $\Theta_{F}^{\mathbf{x}}(u)=\mathbf{f}^{\mathbf{x}} u \mathbf{f}^{-\mathbf{x}}$, where $\mathbf{f}^{\mathbf{x}}:=f_{1}^{x_{1}} \ldots f_{k}^{x_{k}}$. For a $D_{F} \mathcal{U}$-module $N$ by $\Phi_{F}^{\mathbf{x}} N$ we denote the $D_{F} \mathcal{U}$-module $N$ twisted by $\Theta_{F}^{\mathrm{x}}$. The action on $\Phi_{F}^{\mathrm{x}} N$ is given by

$$
u \cdot v^{\mathbf{x}}:=\left(\Theta_{F}^{\mathbf{x}}(u) \cdot v\right)^{\mathbf{x}},
$$

where $u \in D_{F} \mathcal{U}, v \in N$, and $w^{\mathbf{x}}$ stands for the element $w$ considered as an element of $\Phi_{F}^{\mathbf{x}} N$. In the case $\mathbf{x} \in \mathbb{Z}^{k}$, there is a natural isomorphism of $D_{F} \mathcal{U}$-modules $M \rightarrow \Phi_{F}^{\mathbf{x}} M$ given by $m \mapsto\left(\mathbf{f}^{\mathbf{x}} \cdot m\right)^{\mathbf{x}}$ with inverse map defined by $n^{\mathbf{x}} \mapsto \mathbf{f}^{-\mathbf{x}} \cdot n$. In view of this isomorphism, for $\mathbf{x} \in \mathbb{Z}^{k}$, we will identify $M$ with $\Phi_{F}^{\mathbf{x}} M$, and for any $\mathbf{x} \in \mathbb{C}^{k}$ will write $\mathbf{f}^{\mathbf{x}} \cdot m$ (or simply $\mathbf{f}^{\mathbf{x}} m$ ) for $m^{-\mathbf{x}}$ whenever $m \in M$.

The basic properties of the twisting functor $\Phi_{F}^{\mathbf{x}}$ on $D_{F} \mathcal{U}$-mod are summarized in the following lemma. The proofs are straightforward. 
Lemma 7.2. Let $F=\left\{f_{1}, \ldots, f_{k}\right\}$ be a set of locally ad-nilpotent commuting elements of $\mathcal{U}, M$ and $N$ be $D_{F} \mathcal{U}$-modules, $m \in M, u \in \mathcal{U}$, and $\mathbf{x}, \mathbf{y} \in \mathbb{C}^{k}$.

(i) $\Theta_{F}^{\mathbf{x}} \circ \Theta_{F}^{\mathbf{y}}=\Theta_{F}^{\mathbf{x}+\mathbf{y}}$, in particular $\mathbf{f}^{\mathbf{x}} \cdot\left(\mathbf{f}^{\mathbf{y}} \cdot m\right)=\mathbf{f}^{\mathbf{x}+\mathbf{y}} \cdot m$;

(ii) $\Phi_{F}^{\mathbf{x}} \Phi_{F}^{\mathbf{y}}=\Phi_{F}^{\mathbf{x}+\mathbf{y}}$, in particular, $\Phi_{F}^{\mathbf{x}} \Phi_{F}^{-\mathbf{x}}=$ Id on the category of $D_{F} \mathcal{U}$-modules;

(iii) $\mathbf{f}^{\mathbf{x}} \cdot\left(u \cdot\left(\mathbf{f}^{-\mathbf{x}} \cdot m\right)\right)=\Theta_{F}^{\mathbf{x}}(u) \cdot m$;

(iv) $\Phi_{F}^{\mathbf{x}}$ is an exact functor;

(v) $M$ is simple (respectively, injective) if and only if $\Phi_{F}^{\mathbf{x}} M$ is simple (respectively, injective);

(vi) $\operatorname{Hom}_{\mathcal{U}}(M, N)=\operatorname{Hom}_{\mathcal{U}}\left(\Phi_{F}^{\mathbf{x}} M, \Phi_{F}^{\mathbf{x}} N\right)$.

For any $\mathcal{U}$-module $M$, and $\mathbf{x} \in \mathbb{C}^{k}$ we define the twisted localization $D_{F}^{\mathbf{x}} M$ of $M$ relative to $F$ and $\mathbf{x}$ by $D_{F}^{\mathbf{x}} M:=\Phi_{F}^{\mathbf{x}} D_{F} M$. The twisted localization is a exact functor from $\mathcal{U}$-mod to $D_{F} \mathcal{U}$-mod.

7.2. Twisted localization of generalized weight $(\mathcal{U}, \mathcal{H})$-modules. In this subsection we apply the functor $D_{F}^{\mathrm{x}}$ to the category of generalized weight $(\mathcal{U}, \mathcal{H})$-modules.

Lemma 7.3. Assume that $f_{i} \in \mathcal{U}^{\mathbf{a}_{\mathbf{i}}}$ for $\mathbf{a}_{\mathbf{i}} \in \mathbf{Q}$.

(i) If $M$ is a generalized weight $(\mathcal{U}, \mathcal{H})$-module, then $D_{F} M$ is a generalized weight $\left(D_{F} \mathcal{U}, \mathcal{H}\right)$-module.

(ii) If $N$ is a generalized weight $\left(D_{F} \mathcal{U}, \mathcal{H}\right)$-module then $\mathbf{f}^{\mathbf{x}} m \in N^{(\lambda+\mathbf{x a})}$ whenever $m \in N^{(\lambda)}$, where $\mathbf{x a}=x_{1} \mathbf{a}_{\mathbf{1}}+\ldots+x_{k} \mathbf{a}_{\mathbf{k}}$. In particular, $\Phi_{F}^{\mathbf{x}} N$ is a generalized weight $\left(D_{F} \mathcal{U}, \mathcal{H}\right)$-module.

7.3. Twisted localization in $(\mathcal{D}, \mathcal{H})$-mod. Consider now $\mathcal{U}=\mathcal{D}(n+1)$ and $\mathcal{H}=$ $\mathbb{C}\left[t_{0} \partial_{0}, \ldots, t_{n} \partial_{n}\right]$. In this case we set $D_{i}^{+}=D_{\left\{\partial_{i}\right\}}, D_{i}^{-}=D_{\left\{t_{i}\right\}}, D_{i}^{x,-}:=D_{\left\{t_{i}\right\}}^{x}, D_{i}^{x,+}=$ $D_{\left\{\partial_{i}\right\}}^{x}$, and $D_{i, j}^{x}=D_{\left\{t_{i} \partial_{j}\right\}}^{x}$ for $x \in \mathbb{C}$ and $i \neq j$.

Lemma 7.4. Assume that $i \in \operatorname{Int}(\nu)$ and $J \in \mathcal{P}(\nu)$.

If $i \in J$, then

$$
D_{i}^{+}\left(\mathcal{F}_{\nu}^{\log }(J)\right) \simeq \mathcal{F}_{\nu}^{\log }(J), D_{i}^{-}\left(\mathcal{F}_{\nu}^{\log }(J)\right) \simeq \mathcal{F}_{\nu}^{\log }(J \backslash i) .
$$

If $i \notin J$, then

$$
D_{i}^{-}\left(\mathcal{F}_{\nu}^{\log }(J)\right) \simeq \mathcal{F}_{\nu}^{\log }(J), D_{i}^{+}\left(\mathcal{F}_{\nu}^{\log }(J)\right) \simeq \mathcal{F}_{\nu}^{\log }(J \cup i) .
$$

Proof. First, we have the relations

$$
D_{i}^{+} \sigma_{i}=\sigma_{i} D_{i}^{-}, D_{i}^{ \pm} \sigma_{j}=\sigma_{j} D_{i}^{ \pm} \text {if } i \neq j .
$$

Therefore it is sufficient to prove

$$
D_{i}^{-}\left(\mathcal{F}_{\nu}^{\log }\right) \simeq \mathcal{F}_{\nu}^{\log }, D_{i}^{+}\left(\mathcal{F}_{\nu}^{\log }\right) \simeq \mathcal{F}_{\nu}^{\log }(\{i\}) .
$$

Note that $\mathcal{D}(n+1)=\mathcal{D}(n) \otimes D(1)$ and

$$
\mathcal{F}_{\nu}^{\log }=\mathcal{F}_{\nu, i}^{\log } \otimes \mathbb{C}\left[t_{i}, t_{i}^{-1}, \log t_{i}\right]
$$


where $\mathcal{F}_{\nu, i}^{\log }$ is the analogue of $\mathcal{F}_{\nu}^{\log }$ for the corresponding $\mathcal{D}(n)$. Hence we need to prove the latter relation only for $\mathcal{D}(1)$. Note that in this case we may assume $\nu=0$. Then $\mathcal{F}_{\nu}^{\log }(\emptyset)=\mathbb{C}\left[t, t^{-1}, \log t\right]$ and it has an increasing socle filtration

$$
\mathbb{C}\left[t, t^{-1}, \log t\right]_{0}=0 \subset \mathbb{C}\left[t, t^{-1}, \log t\right]_{1} \subset \mathbb{C}\left[t, t^{-1}, \log t\right]_{2} \subset \ldots,
$$

where

$$
\mathbb{C}\left[t, t^{-1}, \log t\right]_{2 i}=\mathbb{C}\left[t, t^{-1}\right] \otimes\left(\sum_{j=0}^{i-1} \mathbb{C}(\log t)^{j}\right), i \geq 1
$$

and

$$
\mathbb{C}\left[t, t^{-1}, \log t\right]_{2 i+1}=\mathbb{C}\left[t, t^{-1}, \log t\right]_{2 i} \oplus \mathbb{C}[t](\log t)^{i}, i \geq 0 .
$$

Note that $\mathbb{C}\left[t, t^{-1}, \log t\right]_{1}$ is the maximal subspace on which $\partial$ acts locally nilpotently. Hence

$$
D_{1}^{+}\left(\mathbb{C}\left[t, t^{-1}, \log t\right]\right)=\mathbb{C}\left[t, t^{-1}, \log t\right] / \mathbb{C}\left[t, t^{-1}, \log t\right]_{1} .
$$

But $\mathbb{C}\left[t, t^{-1}, \log t\right] / \mathbb{C}\left[t, t^{-1}, \log t\right]_{1}$ has a unique simple submodule isomorphic to $\mathcal{S}_{\nu}(\{1\})$.

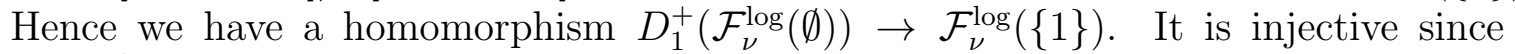
$D_{1}^{+}\left(\mathcal{F}_{\nu}^{\log (\emptyset))}\right.$ has a unique simple submodule which maps to a non-zero space, and it is surjective by comparison of the socle filtration of both modules. Thus, we have $D_{1}^{+}\left(\mathcal{F}_{\nu}^{\log }(\emptyset)\right) \simeq \mathcal{F}_{\nu}^{\log }(\{1\})$

The isomorphism $D_{i}^{-}\left(\mathcal{F}_{\nu}^{\log }\right) \simeq \mathcal{F}_{\nu}^{\log }$ follows from the fact that $t_{i}$ is an invertible operator on $\mathcal{F}_{\nu}^{\log }$.

Recall that $\varepsilon_{i} \in \mathbb{C}^{n+1}$ are defined by $\left(\varepsilon_{i}\right)_{j}=\delta_{i j}$. Lemma 7.4 easily implies the following.

Lemma 7.5. With notations as above

$$
D_{i}^{x,+}\left(\mathcal{F}_{\nu}^{\log }(J)\right) \simeq \mathcal{F}_{\nu-x \varepsilon_{i}}^{\log }(J \cup i), D_{i}^{x,-}\left(\mathcal{F}_{\nu}^{\log }(J)\right) \simeq \mathcal{F}_{\nu+x \varepsilon_{i}}^{\log }(J \backslash i) .
$$

Corollary 7.6. Let $J=\left\{j_{1}, \ldots, j_{k}\right\}$ be a nonempty proper subset of $\{0, \ldots, n\}$, and let $\left\{i_{1}, \ldots, i_{\ell}\right\}=\{0, \ldots, n\} \backslash J$. Then for any $\mu, \nu \in \mathbb{C}^{n+1}$,

$$
D_{i_{1}}^{\nu_{i_{1}}-\mu_{i_{1}},-} \ldots D_{i_{k}}^{\nu_{i_{\ell}}-\mu_{i_{\ell}},-} D_{j_{1}}^{\mu_{j_{1}}-\nu_{j_{1}},+} \ldots D_{j_{k}}^{\mu_{j_{k}}-\nu_{j_{k}},+} \mathcal{F}_{\mu}^{\log } \simeq \mathcal{F}_{\nu}^{\log }(J) .
$$

Lemma 7.7. The following isomorphisms hold for every $M$ in $(\mathcal{D}, \mathcal{H})$-mod, $i \neq j$, and $x, y \in \mathbb{C}$.

(i) $D_{i}^{x, \pm} D_{j}^{y, \pm} M \simeq D_{j}^{y, \pm} D_{i}^{x, \pm} M$.

(ii) $D_{i}^{x, \pm} D_{i}^{y, \pm} M \simeq D_{i}^{x+y, \pm} M$

(iii) $D_{i}^{x,-} D_{j}^{x,+} M \simeq D_{j}^{x,+} D_{i}^{x,-} M \simeq D_{i, j}^{x} M$.

Proof. The isomorphisms can be defined as follows $\left.t_{i}^{x} \cdot\left(t_{j}^{y}\right) \cdot m\right) \mapsto t_{j}^{y} \cdot\left(t_{i}^{x} \cdot m\right)$ and $\left.\partial_{i}^{x} \cdot\left(\partial_{j}^{y}\right) \cdot m\right) \mapsto \partial_{j}^{y} \cdot\left(\partial_{i}^{x} \cdot m\right)$ for (i);

$t_{i}^{x} \cdot\left(t_{i}^{y} \cdot m\right) \mapsto t_{i}^{x+y} \cdot m$ and $\partial_{i}^{x} \cdot\left(\partial_{i}^{y} \cdot m\right) \mapsto \partial_{i}^{x+y} \cdot m$ for (ii);

$t_{i}^{x} \cdot\left(\partial_{j}^{x} \cdot m\right) \mapsto\left(t_{i} \partial_{j}\right)^{x} \cdot m$ for (iii). 
Corollary 7.8. Let $\mu, \nu \in \mathbb{C}^{n+1}$ be such that $\mu_{0}+\ldots+\mu_{n}=\nu_{0}+\ldots+\nu_{n}$. For any proper nonempty subset $J$ of $\{0, \ldots, n\}$, there is a subset $S_{J}$ of $\{(i, j) \mid i \notin J, j \in J\}$ with the following properties

(i) $S_{J}$ consists of $n$ elements;

(ii) For every $i \notin J$ (respectively, $j \in J$ ), there exists $j \in J$ (respectively, $i \notin J$ ) such that $(i, j) \in S_{J}$;

(iii)

$$
\prod_{(i, j) \in S_{J}} D_{i, j}^{z(i, j)} \mathcal{F}_{\mu}^{\log } \simeq \mathcal{F}_{\nu}^{\log }(J)
$$

for some $z(i, j) \in \mathbb{C}$ (note that the functors $D_{i, j}^{z(i, j)}$ in the above product commute due to Lemma 7.7).

Proof. Let $J=\left\{j_{1}, \ldots, j_{k}\right\}$ and $\left\{i_{1}, \ldots, i_{\ell}\right\}$ is the complement of $J$ in $\{0,1, \ldots, n\}$. Consider the system

$$
\begin{aligned}
& \sum_{s=1}^{k} z(r, s)=\nu_{i_{r}}-\mu_{i_{r}}, r=1, \ldots, \ell \\
& \sum_{r=1}^{\ell} z(r, s)=\mu_{j_{s}}-\nu_{j_{s}}, s=1, \ldots, k
\end{aligned}
$$

We can find a solution of the above system such that the set $S_{J}^{\prime}$ of nonzero $z(i, j)$ satisfy conditions (i) and (ii) of the corollary. To complete the proof we use the isomorphism in Corollary 7.6 and the preceding lemma.

7.4. Twisted localization in $\mathcal{G B}$. In this case we consider $\mathcal{U}=U(\mathfrak{s l}(n+1))$ and $\mathcal{H}=S(\mathfrak{h})$. The multiplicative sets will be always of the form $F=\left\langle e_{\alpha} \mid \alpha \in \Gamma\right\rangle$, where $\Gamma$ is a set of $k$ commuting roots and $e_{\alpha}$ is in the $\alpha$-root space of $\mathfrak{s l}(n+1)$. For $\mathbf{x} \in \mathbb{C}^{k}$, we write $D_{\Gamma}$ and $D_{\Gamma}^{\mathbf{x}}$ for $D_{F}$ and $D_{F}^{\mathrm{x}}$, respectively.

Recall that a subset $\Gamma$ of $\Delta$ is a set of commuting roots if $\alpha, \beta \in \Gamma$ imply $\left[e_{\alpha}, e_{\beta}\right]=0$. The maximal (with respect to inclusion) sets of commuting roots can be parametrized by the set $\mathcal{P}_{-1}$ (cf. Section 6.1) of nonempty proper subsets of $\{0,1, \ldots, n\}$. Indeed, for $J=\left\{i_{1}, \ldots, i_{k}\right\}$ in $\mathcal{P}_{-1}$,

$$
\Lambda_{J}:=\left\{\varepsilon_{i}-\varepsilon_{j} \mid i \in J, j \notin J\right\}
$$

is a maximal set of commuting roots. Then one can check that the correspondence $J \mapsto \Lambda_{J}$ is a bijection between $\mathcal{P}_{-1}$ and the set of all maximal commuting sets of roots. In particular, if $\Gamma$ is a set of $n$ linearly independent commuting roots then there is a unique $J \subset \mathcal{P}_{-1}$ such that $\Gamma \subset \Lambda_{J}$.

The following theorem is proven in [19].

Proposition 7.9. Every simple module in $\mathcal{G B}$ (equivalently, in $\mathcal{B}$ ) is isomorphic to $D_{\Gamma}^{\mathrm{x}} L$ for some simple highest weight module $L$ in $\mathcal{G B}$, a set $\Gamma$ of $n$ commuting roots, and $\mathbf{x} \in \mathbb{C}^{n}$. 


\section{Equivalence of CATEgories of generalized Weight $\mathcal{D}^{E}$-MOdules AND GENERALIZED BOUNDED $\mathfrak{s l}(n+1)$-MODULES}

8.1. The functor $\Psi$. Consider the homomorphism $\psi: U(\mathfrak{s l}(n+1)) \rightarrow \mathcal{D}(n+1)$ defined by $\psi\left(E_{i j}\right)=t_{i} \partial_{j}$. The image of $\psi$ is contained in $\mathcal{D}^{E}$. Using lift by $\psi$ any $\mathcal{D}^{E}$-module becomes $\mathfrak{s l}(n+1)$-module. Since $\psi(U(\mathfrak{h})) \subset \mathcal{H}$, one has a functor $\Psi:\left(\mathcal{D}^{E}, \mathcal{H}\right)-\bmod \rightarrow \overline{\mathcal{G B}}$. Obviously $\Psi$ is exact.

In all statements below we assume that $\nu \in \mathbb{C}^{n+1}$.

Lemma 8.1. Let $|\nu|=a$. Then

$$
\Psi\left({ }^{\mathrm{b}}\left(\mathcal{D}^{E}, \mathcal{H}\right)_{\nu}^{a}-\bmod \right) \subset \mathcal{G} \mathcal{B}_{\gamma(\nu)}^{\gamma\left(a \varepsilon_{0}\right)}
$$

Proof. The inclusion

$$
\Psi\left({ }^{\mathrm{b}}\left(\mathcal{D}^{E}, \mathcal{H}\right)_{\nu}-\bmod \right) \subset \mathcal{G} \mathcal{B}_{\gamma(\nu)}
$$

follows immediately from the definition. Since $\psi(Z(U(\mathfrak{s l}(n+1))))$ is contained in the center of $\mathcal{D}^{E}$ and the latter is generated by $E, \Psi(M)$ admit the same central character for all $M \in^{\mathrm{b}}\left(\mathcal{D}^{E}, \mathcal{H}\right)^{a}-\bmod$. Furthermore, $\Psi\left(S_{a \varepsilon_{0}}(\emptyset)\right)$ is a highest weight module with highest weight $a \varepsilon_{0}$. Therefore

$$
\Psi\left({ }^{\mathrm{b}}\left(\mathcal{D}^{E}, \mathcal{H}\right)_{\nu}^{a}-\bmod \right) \subset \mathcal{G B}_{\gamma(\nu)}^{\gamma\left(a \varepsilon_{0}\right)}
$$

In the case $\Gamma=\{\alpha\}$ we will use the following notation: $D_{\alpha}^{x}=D_{\left\{e_{\alpha}\right\}}^{x}$ for $x \in \mathbb{C}$.

Lemma 8.2. If $M$ is a module in $\left(\mathcal{D}^{E}, \mathcal{H}\right)-\bmod$ and $x \in \mathbb{C}$, then $D_{\varepsilon_{i}-\varepsilon_{j}}^{x} \Psi(M) \simeq$ $\Psi\left(D_{i, j}^{x} M\right)$.

Proof. The map $e_{\varepsilon_{i}-\varepsilon_{j}}^{x} \cdot m \mapsto\left(t_{i} \partial_{j}\right)^{x} \cdot m$ provides the desired isomorphism.

Remark 8.3. Using the above lemma and the fact that the functor $\Psi:{ }^{\mathrm{b}}\left(\mathcal{D}^{E}, \mathcal{H}\right)-\bmod \rightarrow$ $\mathcal{G B}$ is surjective (which follows from Theorem 8.7), we can prove that for $\Gamma=$ $\left\{\alpha_{1}, \ldots, \alpha_{k}\right\}$, the localization functor $D_{\Gamma}^{\mathbf{x}}$ on $\mathcal{G B}$ depends only on Span $\Gamma$ and the weight $\sum_{i=1}^{k} x_{i} \alpha_{i}$. More precisely, if $\Gamma^{\prime}=\left\{\beta_{1}, \ldots, \beta_{\ell}\right\}$ is another set of commuting roots of $\mathfrak{s l}(n+1)$ such that $\operatorname{Span}_{\mathbb{C}} \Gamma=\operatorname{Span}_{\mathbb{C}} \Gamma^{\prime}$ and if $\mathbf{y} \in \mathbb{C}^{\ell}$ be such that $\sum_{i=1}^{k} x_{i} \alpha_{i}=\sum_{i=1}^{\ell} y_{i} \beta_{i}$, then $D_{\Gamma}^{\mathbf{x}} M \simeq D_{\Gamma^{\prime}}^{\mathbf{y}} M$ for every $M$ in $\mathcal{G} B$.

Here is a short proof of this statement. Denote $I=\left\{i \mid \varepsilon_{i}-\varepsilon_{j} \in \Gamma\right.$, for some $\left.j\right\}$, and $J=\left\{j \mid \varepsilon_{i}-\varepsilon_{j} \in \Gamma\right.$, for some $\left.i\right\}$ and define $I^{\prime}$ and $J^{\prime}$ in a similar way from $\Gamma^{\prime}$. Then $\operatorname{Span}_{\mathbb{C}} \Gamma=\operatorname{Span}_{\mathbb{C}} \Gamma^{\prime}$ implies that $I=I^{\prime}$ and $J=J^{\prime}$. Let now $M=\Psi(N)$. Applying multiple times Lemma 7.7 and Lemma 8.2 we obtain that

$$
D_{\Gamma}^{\mathrm{x}} M \simeq D_{\Gamma}^{\mathrm{x}} \Psi(N) \simeq \Psi\left(\prod_{i \in I} D_{i}^{z_{i},+} \prod_{j \in J} D_{j}^{z_{j},-}(N)\right) \simeq D_{\Gamma^{\prime}}^{\mathrm{y}} \Psi(N) \simeq D_{\Gamma^{\prime}}^{\mathrm{y}} M,
$$

where $\sum_{k} x_{k} \alpha_{k}=\sum_{i \in I} z_{i} \varepsilon_{i}-\sum_{j \in J} z_{j} \varepsilon_{j}$. 


\subsection{The generic case.}

Lemma 8.4. Suppose $a \notin \mathbb{Z}$, or $a=-1, \ldots,-n$ for $n \geq 2$ ( $a$ is arbitrary for $n=1$ ). Any simple $S \in \mathcal{G B}^{\gamma\left(a \varepsilon_{0}\right)}$ is isomorphic to $\Psi\left(S^{\prime}\right)$ for some simple $S^{\prime} \in{ }^{\mathrm{b}}\left(\mathcal{D}^{E}, \mathcal{H}\right)_{\nu}^{a}-\bmod$.

Proof. By Proposition 7.9, every simple $S$ in $\mathcal{G B}^{\gamma\left(a \varepsilon_{0}\right)}$ is isomorphic to $D_{\Gamma}^{\mathrm{x}} L$, where $L$ is a simple highest weight module in $\mathcal{G B}^{\gamma\left(a \varepsilon_{0}\right)}, \Gamma$ is a commuting set of roots and $\mathbf{x} \in \mathbb{C}^{n}$. As mentioned in the proof of Lemma 8.1, $L=\Psi\left(S_{a \varepsilon_{0}}(\emptyset)\right)$. Therefore the annihilators in $U(\mathfrak{s l}(n+1))$ of all simple objects $S$ in $\mathcal{G B}^{\gamma\left(a \varepsilon_{0}\right)}$ coincide. So, every such $S$ is annihilated by the kernel of $\psi$, and thus $S=\Psi\left(S^{\prime}\right)$ for some $S^{\prime} \in$ $\mathrm{b}\left(\mathcal{D}^{E}, \mathcal{H}\right)_{\nu}^{a}-\bmod$.

We use the notation $\mathbb{C}^{k}\left[u_{0}, \ldots, u_{n}\right]$ and $\mathbb{C}^{<k}\left[u_{0}, \ldots, u_{n}\right]$ for the polynomials of degree $k$, and of degree strictly less than $k$, respectively.

Lemma 8.5. Let $\operatorname{Int}(\nu)=\emptyset, n \geq 2$ or $a \neq-1$.

(a) $\Psi\left(\Gamma_{a}\left(\mathcal{F}_{\nu}\right)\right)$ is the unique simple submodule of $\Psi\left(\Gamma_{a}\left(\mathcal{F}_{\nu}^{\log }\right)\right)$.

(b) For any positive $k$

$$
\operatorname{soc}^{k} \Psi\left(\Gamma_{a}\left(\mathcal{F}_{\nu}^{\log }\right)\right) \simeq \Psi\left(\Gamma_{a}\left(\mathcal{F}_{\nu}^{\log ,<k}\right)\right),
$$

where $\mathcal{F}_{\nu}^{\log ,<k}=\mathcal{F}_{\nu} \otimes \mathbb{C}^{<k}\left[u_{0}, \ldots, u_{n}\right]$.

Proof. (a) Assume that $\Psi\left(\Gamma_{a}\left(\mathcal{F}_{\nu}^{\text {log }}\right)\right)$ has a simple submodule $M$ distinct from $\Psi\left(\Gamma_{a}\left(\mathcal{F}_{\nu}\right)\right)$.

Every simple module in $\mathcal{G B}_{\gamma(\nu)}^{\gamma\left(a \varepsilon_{0}\right)}$ is a weight module. Let us fix a weight vector $m=f\left(u_{0}, \ldots, u_{n}\right) t_{0}^{\lambda_{0}} \ldots t_{n}^{\lambda_{n}}$ in $M$, where $f\left(u_{0}, \ldots, u_{n}\right) \in \mathbb{C}\left[u_{0}, \ldots, u_{n}\right]$ and $u_{i}=\log t_{i}$. Since $t_{i} \partial_{i}-t_{j} \partial_{j}$ act as a scalar multiple on $m$ we easily conclude that $f\left(u_{0}, \ldots, u_{n}\right)=$ $g\left(u_{0}+\ldots+u_{n}\right)$ for some polynomial $g$. Thus, we have that

$$
S_{\mathfrak{h}}\left(\Psi\left(\Gamma_{a}\left(\mathcal{F}_{\nu}^{\log }\right)\right)\right)=\Psi\left(\Gamma_{a}\left(\mathcal{F}_{\nu} \otimes \mathbb{C}[u]\right)\right),
$$

where $u=u_{0}+\ldots+u_{n}$ and $S_{\mathfrak{h}}$ stands for the functor $\mathcal{G B}^{\gamma\left(a \varepsilon_{0}\right)} \rightarrow \mathcal{B}^{\gamma\left(a \varepsilon_{0}\right)}$ mapping a module to its submodule consisting of all $\mathfrak{h}$-eigenvectors. Now, the statement follows from Lemma 5.4 of $[18$.

(b) We apply induction on $k$. The base case $k=1$ follows from part (a). Assume that the statement holds for all $k<\ell$. In the case $k=\ell$ consider the quotient $N=\Psi\left(\Gamma_{a}\left(\mathcal{F}_{\nu}^{\log }\right)\right) / \operatorname{soc}^{\ell} \Psi\left(\Gamma_{a}\left(\mathcal{F}_{\nu}^{\log }\right)\right)$. By the same argument as in (a)

$$
S_{\mathfrak{h}}(N)=\Psi\left(\Gamma_{a}\left(\mathcal{F}_{\nu} \otimes \mathbb{C}^{\ell}\left[u_{0}, \ldots, u_{n}\right] \otimes \mathbb{C}[u]\right)\right) .
$$

Therefore $S_{\mathfrak{h}}(N)$ is isomorphic to the direct sum of $\left(\begin{array}{c}n+k \\ k\end{array}\right)$ copies of $\Psi\left(\Gamma_{a}\left(\mathcal{F}_{\nu} \otimes \mathbb{C}[u]\right)\right)$ and the socle of $S_{\mathfrak{h}}(N)$ coincides with $\Psi\left(\Gamma_{a}\left(\mathcal{F}_{\nu} \otimes \mathbb{C}^{\ell}\left[u_{0}, \ldots, u_{n}\right]\right)\right)$ by (a).

Remark 8.6. Let us explain why Lemma 8.5 is false in the case $n=1, a=-1$. Indeed, let $\nu=c \varepsilon_{0}+(-1-c) \varepsilon_{1}$ for some $c \in \mathbb{C} \backslash \mathbb{Z}$. Let $\varphi: c+\mathbb{Z} \rightarrow \mathbb{C}$ be a function satisfying the condition $\varphi(x+1)-\varphi(x)=\frac{-1}{x+1}$ for all $x \in c+\mathbb{Z}$. Then it is easy to check that the elements $t_{0}^{x} t_{1}^{-1-x} \log t_{0} t_{1}+\varphi(x) t_{0}^{x} t_{1}^{-1-x}$ for $x \in c+\mathbb{Z}$ span a 
simple submodule of $\Psi\left(\Gamma_{a}\left(\mathcal{F}_{\nu}^{\log }\right)\right)$. On the other hand, $\Psi\left(\Gamma_{a}\left(\mathcal{F}_{\nu}\right)\right)$ is another simple submodule in $\Psi\left(\Gamma_{a}\left(\mathcal{F}_{\nu}^{\log }\right)\right)$. Hence $\Psi\left(\Gamma_{a}\left(\mathcal{F}_{\nu}^{\log }\right)\right)$ does not have a simple socle.

Theorem 8.7. Assume that $a \notin \mathbb{Z}$ or $a=-1, \ldots,-n$ for $n \geq 2$ and $a \neq-1$ for $n=1$. Then $\Psi$ provides equivalence between ${ }^{\mathrm{b}}\left(\mathcal{D}^{E}, \mathcal{H}\right)^{a}-\bmod$ and $\mathcal{G B}^{\gamma\left(a \varepsilon_{0}\right)}$ and between $\left(\mathcal{D}^{E}, \mathcal{H}\right)^{a}-\bmod$ and $\overline{\mathcal{G B}}^{\gamma\left(a \varepsilon_{0}\right)}$.

Moreover, $\Psi$ provides equivalence between ${ }_{\mathrm{s}}^{\mathrm{b}}\left(\mathcal{D}^{E}, \mathcal{H}\right)^{a}-\bmod$ and $\mathcal{B}^{\gamma\left(a \varepsilon_{0}\right)}-\bmod$, as well as, between ${ }_{\mathrm{s}}\left(\mathcal{D}^{E}, \mathcal{H}\right)^{a}-\bmod$ and $\overline{\mathcal{B}}^{\gamma\left(a \varepsilon_{0}\right)}-\bmod$.

Proof. We prove the theorem in several steps. First, we prove it for the cuspidal blocks.

Lemma 8.8. Assume that $a \notin \mathbb{Z}$ or $a=-1, \ldots,-n$ for $n \geq 2$ and $a \neq-1$ for $n=1$. Let $\operatorname{Int}(\nu)=\emptyset$.

(a) $\Psi\left(\Gamma_{a}\left(\mathcal{F}_{\nu}^{\log }\right)\right)$ is an indecomposable injective in $\overline{\mathcal{G B}}$.

(b) $\Psi$ provides equivalence between ${ }^{\mathrm{b}}\left(\mathcal{D}^{E}, \mathcal{H}\right)_{\nu}^{a}-\bmod$ and $\mathcal{G B}_{\gamma(\nu)}^{\gamma\left(a \varepsilon_{0}\right)}$, and between $\left(\mathcal{D}^{E}, \mathcal{H}\right)_{\nu}^{a}-\bmod$ and $\overline{\mathcal{G B}}_{\gamma(\nu)}^{\gamma\left(a \varepsilon_{0}\right)}$.

Proof. (a) Both categories $\left(\mathcal{D}^{E}, \mathcal{H}\right)_{\nu}^{a}-\bmod$ and $\overline{\mathcal{G B}}_{\gamma(\nu)}^{\gamma\left(a \varepsilon_{0}\right)}$ have one up to isomorphism simple module $S=\Gamma_{a}\left(\mathcal{F}_{\nu}\right)$ and $\Psi(S)$, respectively. As follows from [20], $\overline{\mathcal{G B}}_{\gamma(\nu)}^{\gamma\left(a \varepsilon_{0}\right)}$ is equivalent to the category of locally nilpotent $\mathbb{C}\left[z_{0}, \ldots, z_{n}\right]$-modules. Let $J$ be the indecomposable injective in $\overline{\mathcal{G B}}_{\gamma(\nu)}^{\gamma\left(a \varepsilon_{0}\right)}$. The length of $\operatorname{soc}^{k}(J)$ is the same as the length of $\operatorname{soc}^{k}\left(\Psi\left(\Gamma_{a}\left(\mathcal{F}_{\nu}^{\log }\right)\right)\right)$. On the other hand, we have an injective homomorphism $j: \Psi\left(\Gamma_{a}\left(\mathcal{F}_{\nu}^{\log }\right)\right) \rightarrow J$ induced by the embedding $\Psi(S) \rightarrow J$. Since the terms of the socle filtration of $J$ and $\Psi\left(\Gamma_{a}\left(\mathcal{F}_{\nu}^{\log }\right)\right)$ have the same length, $j$ is an isomorphism.

Part (b) follows from (a).

Lemma 8.9. Assume that $\nu \in \mathbb{C}^{n+1}$ and let $J \in \mathcal{P}(\nu)$ be such that $J \neq \emptyset,\{0, \ldots, n\}$ whenever $a \in \mathbb{Z}$ and $\nu \in \mathbb{Z}^{n+1}$. Then the functor $\operatorname{Hom}_{\mathfrak{g}}\left(\cdot, \Psi\left(\Gamma_{a}\left(\mathcal{F}_{\nu}^{\log }(J)\right)\right)\right)$ is exact on modules in the category $\mathcal{G B}^{\gamma\left(a \varepsilon_{0}\right)}$.

Proof. Let us first fix $\mu$ with $\operatorname{Int}(\mu)=\emptyset$ and such that $\mu_{0}+\ldots+\mu_{n}=\nu_{0}+\ldots+\nu_{n}$. We use Corollary 7.8 and find a subset $S_{J}$ of $\{(i, j) \mid i \notin J, j \in J\}$ that satisfy (i)-(iii) of that corollary. In particular,

$$
\mathcal{F}_{\nu}^{\log }(J) \simeq \prod_{(i, j) \in S_{J}} D_{i, j}^{z(i, j)} \mathcal{F}_{\mu}^{\log }
$$

where $z(i, j)$ are such that $\sum_{s=1}^{\ell} z(r, s)=\nu_{i_{r}}-\mu_{i_{r}}, \sum_{r=1}^{k} z(r, s)=\mu_{j_{s}}-\nu_{j_{s}}$. The conditions (i) and (ii) of orollary 7.8 imply that $\Sigma_{J}:=\left\{\varepsilon_{i}-\varepsilon_{j} \mid(i, j) \in S_{J}\right\}$ is a set of $n$ linearly independent commuting roots of $\mathfrak{s l}(n+1)$. Also, as one can easily show, the functor $\Gamma_{a}$ commutes with the twisted localization functors $D_{i, j}^{x}$. Hence

$$
\Psi\left(\Gamma_{a}\left(\prod_{(i, j) \in S_{J}} D_{i, j}^{z(i, j)} \mathcal{F}_{\mu}^{\log }\right)\right) \simeq \Psi\left(\prod_{(i, j) \in S_{J}} D_{i, j}^{z(i, j)}\left(\Gamma_{a}\left(\mathcal{F}_{\mu}^{\log }\right)\right)\right) \simeq D_{\Sigma_{J}}^{\mathrm{x}}\left(\Psi\left(\Gamma_{a}\left(\mathcal{F}_{\mu}^{\log }\right)\right)\right)
$$


where $\nu-\mu=\sum_{i=1}^{n} x_{i} \alpha_{i}$ and $\Sigma_{J}=\left\{\alpha_{1}, \ldots, \alpha_{n}\right\}$. The last isomorphism follows from the identities for $z(r, s)$ and Lemma 8.2. Therefore

$$
\Psi\left(\Gamma_{a}\left(\mathcal{F}_{\nu}^{\log }(J)\right)\right)=D_{\Sigma_{J}}^{\mathbf{x}}\left(\Psi\left(\Gamma_{a}\left(\mathcal{F}_{\mu}^{\log }\right)\right)\right) .
$$

Then using Lemma 7.1 (iv) and Lemma 7.2 (vi) we have

$$
\begin{gathered}
\operatorname{Hom}_{\mathfrak{g}}\left(X, D_{\Sigma_{J}}^{\mathrm{x}} \Psi\left(\Gamma_{a}\left(\mathcal{F}_{\mu}^{\log }\right)\right)\right) \simeq \operatorname{Hom}_{\mathfrak{g}}\left(D_{\Sigma_{J}}(X), D_{\Sigma_{J}}^{\mathrm{x}} \Psi\left(\Gamma_{a}\left(\mathcal{F}_{\mu}^{\log }\right)\right)\right) \simeq \\
\operatorname{Hom}_{\mathfrak{g}}\left(D_{\Sigma_{J}}^{\mathrm{x}}(X), \Psi\left(\Gamma_{a}\left(\mathcal{F}_{\mu}^{\log }\right)\right)\right) .
\end{gathered}
$$

Since $D_{\Sigma_{J}}^{\mathrm{x}}(\cdot)$ is exact, the statement follows from Lemma 8.8(a).

Now we are ready to complete the proof of Theorem 8.7. Note first that any simple $S \in \mathcal{G B}^{\gamma\left(a \varepsilon_{0}\right)}$ is isomorphic to $\Psi\left(S^{\prime}\right)$ for some simple $S^{\prime} \in{ }^{\mathrm{b}}\left(\mathcal{D}^{E}, \mathcal{H}\right)^{a}-\bmod$ by Lemma 8.4.

Next, by Lemma 8.9, $\Psi$ maps indecomposable injectives to indecomposable injectives. Hence Theorem 8.7 follows from Theorem 5.1, The statement for weight modules can be easily obtained by applying the functors $S_{\mathcal{H}^{\prime}}$ and $S_{\mathfrak{h}}$.

8.3. The singular block for $\mathfrak{s l}(2)$-modules. Now we consider the case $n=1$ and $a=-1$.

Proposition 8.10. Let $n=1$. The category $\mathcal{G B}_{\gamma\left(-\varepsilon_{0}\right)}^{\gamma\left(-\varepsilon_{0}\right)}$ is equivalent to the category of nilpotent representation of the quiver

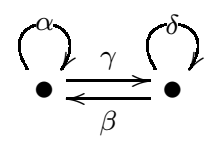

with relations $\alpha \beta \gamma=\beta \gamma \alpha$ and $\delta \gamma \beta=\gamma \beta \delta$, and the category $\mathcal{B}_{0}^{\gamma\left(-\varepsilon_{0}\right)}$ is equivalent to the category of nilpotent representation of the quiver

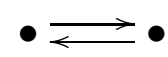

Moreover, the former category is wild, while the latter is tame.

Proof. First, consider the cuspidal block $\overline{\mathcal{G B}}_{\gamma(\nu)}^{\gamma\left(-\varepsilon_{0}\right)}$. This block has only one (up to isomorphism) simple object and one indecomposable injective $J$. As follows from [20], $\operatorname{End}_{\mathfrak{s l}(2)}(J)=\mathbb{C}\left[\left[z_{0}, z_{1}\right]\right]$. On the other hand, $\overline{\mathcal{G B}}_{\gamma\left(-\varepsilon_{0}\right)}^{\gamma\left(-\varepsilon_{0}\right)}$ has two simple modules $S_{+}$and $S_{-}$and therefore two indecomposable injectives $J_{+}$and $J_{-}$. Note that $S_{ \pm}$ are Verma modules with respect to the two opposite Borel subalgebras of $\mathfrak{s l}(2)$. It is an easy exercise to check that

$$
\operatorname{Ext}_{\mathcal{G B}}^{1}\left(S_{+}, S_{+}\right)=\operatorname{Ext}_{\mathcal{G B}}^{1}\left(S_{+}, S_{-}\right)=\operatorname{Ext}_{\mathcal{G B}}^{1}\left(S_{-}, S_{-}\right)=\operatorname{Ext}_{\mathcal{G B}}^{1}\left(S_{-}, S_{+}\right)=\mathbb{C}
$$

This already implies that the quiver of $\mathcal{G B}_{\gamma\left(-\varepsilon_{0}\right)}^{\gamma\left(-\varepsilon_{0}\right)}$ is as above.

To find the relations, we use the fact that $J_{ \pm}=D_{ \pm \alpha}^{x} J$ where $\gamma\left(\varepsilon_{0}\right)-\nu= \pm x \alpha(\mathrm{cf}$. Lemma $7.2(\mathrm{v}))$. Thus, by Lemma 7.2 (vi)

$$
\operatorname{End}_{\mathfrak{s l}(2)}\left(J_{ \pm}\right) \simeq \operatorname{End}_{\mathfrak{s l}(2)}(J) \simeq \mathbb{C}\left[\left[z_{0}, z_{1}\right]\right]
$$


By (8.1) we have $\beta \gamma \in \operatorname{End}_{\mathfrak{s l}(2)}\left(J_{+}\right)=\mathbb{C}\left[\left[z_{0}, z_{1}\right]\right]$. Therefore after a suitable change of variables, without loss of generality, we may assume $\beta \gamma=z_{0}$. Let $\alpha=z_{1}$. Then we have the relation $\alpha \beta \gamma=\beta \gamma \alpha$. We define $\delta$ and obtain the second relation in the same way. Clearly, there are no other relations.

To see that the category $\mathcal{G B}_{\gamma\left(-\varepsilon_{0}\right)}^{\gamma\left(-\varepsilon_{0}\right)}$ is wild set $\beta=0$. Then, the corresponding quotient algebra is the algebra of the following wild quiver without relations.

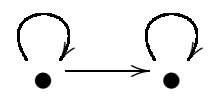

To prove the statement for $\mathcal{B}_{\gamma\left(-\varepsilon_{0}\right)}^{\gamma\left(-\varepsilon_{0}\right)}$ we first observe that it has the same simple objects as $\mathcal{G} \mathcal{B}_{\gamma\left(-\varepsilon_{0}\right)}^{\gamma\left(-\varepsilon_{0}\right)}$ and

$$
\operatorname{Ext}_{\mathcal{B}}^{1}\left(S_{+}, S_{+}\right)=\operatorname{Ext}_{\mathcal{B}}^{1}\left(S_{-}, S_{-}\right)=0, \operatorname{Ext}_{\mathcal{B}}^{1}\left(S_{+}, S_{-}\right)=\operatorname{Ext}_{\mathcal{B}}^{1}\left(S_{-}, S_{+}\right)=\mathbb{C} .
$$

If we denote by $J_{ \pm}^{\prime}$ the injective hulls of $S_{ \pm}$in $\overline{\mathcal{B}}_{\gamma\left(-\varepsilon_{0}\right)}^{\gamma\left(-\varepsilon_{0}\right)}$, then $\operatorname{End}_{\mathfrak{s l}(2)}\left(J_{ \pm}^{\prime}\right) \simeq \mathbb{C}[z]$ by [18] and Lemma 7.2 (vi) as above. In this way we obtain the second quiver with no relations, that is a well-known tame quiver.

\section{Appendix: Some useful commutative diagrams}

Diagram 1.

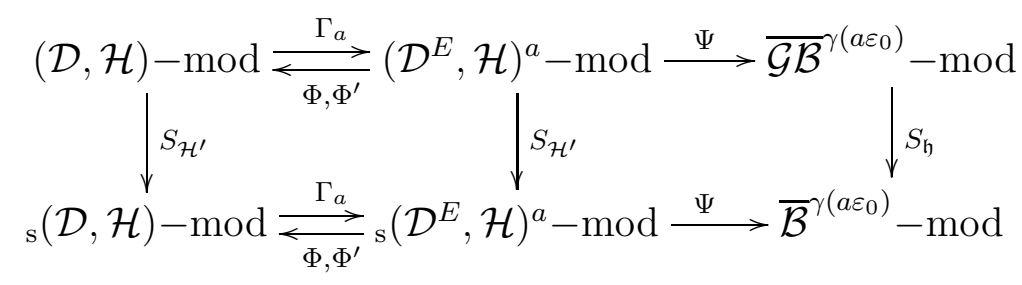

Diagram 2.

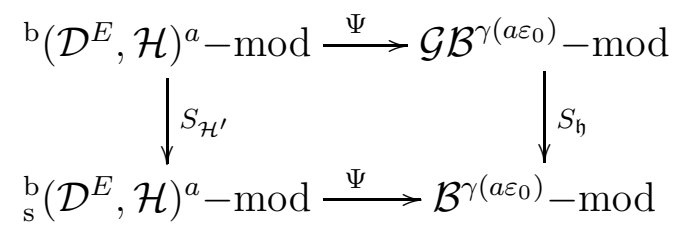

Diagram 3.

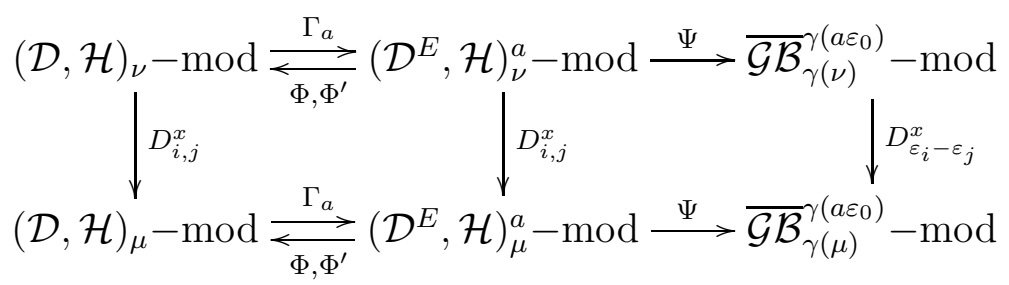


where $\mu=\nu+x\left(\varepsilon_{i}-\varepsilon_{j}\right)$.

\section{REFERENCES}

1. V. Bavula, Generalized Weyl algebras and their representations. Algebra i Analiz 4 (1992), $75-97$.

2. V. Bavula and V. Bekkert, Indecomposable representations of generalized Weyl algebras, Comm. Algebra 28 (2000), 5067-5100.

3. A. Beilinson and J. N. Bernstein, Localisation de g-modules, C. R. Acad. Sci. Ser. A-B 292 (1981), 15-18.

4. A. Beilinson, V. Ginzburg, W. Soergel, Koszul duality patterns in representation theory. $J$. Amer. Math. Soc. 9 (1996), 473-527.

5. V. Bekkert, G. Benkart, V. Futorny, Weight modules for Weyl algebras, in "Kac-Moody Lie algebras and related topics", Contemp. Math. 343 Amer. Math. Soc., Providence, RI, 2004, $17-42$.

6. V. Bekkert, E. N. Marcos, H. A. Merklen, Indecomposables in Derived Categories of SkewedGentle Algebras, Comm. Algebra, Vol. 31, (2003), pp. 2615-2654.

7. G. Benkart, D. Britten, F. Lemire, Modules with bounded weight multiplicities for simple Lie algebras. Math. Z. 225 (1997), 333-353.

8. J. Bernstein, S. Gelfand, Tensor products of finite and infinite-dimensional representations of semisimple Lie algebras, Compositio Math. 41 (1980), 245-285.

9. D. Britten, F. Lemire, A classification of simple Lie modules having a 1-dimensional weight space. Trans. Amer. Math. Soc. 299 (1987), 683-697.

10. D. Britten, O. Khomenko, F. Lemire, V. Mazorchuk, Complete reducibility of torsion free $C_{n^{-}}$ modules of finite degree, J. Algebra 276 (2004), 129-142.

11. P. Donovan, M.R. Freislich, The representation theory of finite graphs and associated algebras. Carleton Mathematical Lecture Notes, No. 5. Carleton University, Ottawa, Ont., 1973.

12. K. Erdmann, Blocks of Tame Representation Type and Related Algebras, Lecture Notes in Math. 1428, Springer-Verlag, New York, 1990.

13. S. Fernando, Lie algebra modules with finite-dimensional weight spaces I, Trans. Amer. Math. Soc. 322 (1990), 757-781.

14. V. Futorny, The weight representations of semisimple finite-dimensional Lie algebras, $\mathrm{Ph}$. D. Thesis, Kiev University, 1987.

15. P. Gabriel, The universal cover of a representation-finite algebra. Representations of algebras (Puebla, 1980), pp. 68V105, Lecture Notes in Math., 903, Springer, Berlin-New York, 1981.

16. Ch. Geiss, J. A. dela Pena, Auslander-Reiten components for clans, Bol. Soc. Mat. Mexicana (3) 5 (1999), 307-326.

17. D. Grantcharov, V. Serganova, Category of $\mathfrak{s p}(2 n)$-modules with bounded weight multiplicities, Mosc. Math. J. 6 (2006), 119-134.

18. D. Grantcharov, V. Serganova, Cuspidal representations of $\mathfrak{s l}(n+1)$, Adv. Math. 224 (2010), $1517-1547$.

19. O. Mathieu, Classification of irreducible weight modules, Ann. Inst. Fourier 50 (2000), 537-592.

20. V. Mazorchuk, C. Stroppel, Cuspidal $\mathfrak{s l}_{n}$-modules and deformations of certain Brauer tree algebras, Adv. Math. 228 (2011) 1008-1042.

21. J. McConnell, J. Robson, Noncommutative Noetherian rings. With the cooperation of L. W. Small. Revised edition. Graduate Studies in Mathematics, 30. American Mathematical Society, Providence, RI, 2001.

22. L. A. Nazarova, Representations of quivers of infinite type. (Russian) Izv. Akad. Nauk SSSR Ser. Mat. 37 (1973), 752-791. 
23. L. Unger, The concealed algebras of the minimal wild hereditary algebras, Bayreuth. Math. Schr. 31 (1990) 145-154.

Dept. of Mathematics, University of Texas at Arlington, Arlington, TX 76019 E-mail address: grandim@uta.edu

Dept. of Mathematics, University of California at Berkeley, Berkeley, CA 94720 E-mail address: serganov@math.berkeley.edu 